\title{
EXISTENCE, UNIQUENESS AND PARAMETRIZATION OF LAGRANGIAN INVARIANT SUBSPACES
}

\author{
GERHARD FREILING* ${ }^{*}$ VOLKER MEHRMANN ${ }^{\dagger}$, AND HONGGUO XU $\ddagger$
}

\begin{abstract}
The existence, uniqueness and parametrization of Lagrangian invariant subspaces for Hamiltonian matrices is studied. Necessary and sufficient conditions and a complete parameterization are given.

Some necessary and sufficient conditions for the existence of Hermitian solutions of algebraic Riccati equations follow as simple corollaries.
\end{abstract}

Keywords. eigenvalue problem, Hamiltonian matrix, symplectic matrix, Lagrangian invariant subspace, algebraic Riccati equation AMS subject classification. 65F15, 93B40, 93B36, 93C60

1. Introduction. The computation of invariant subspaces of Hamiltonian matrices is an important task in many applications in LQ and $H_{\infty}$ control, Kalman filtering or spectral factorization, see $[13,15,20,28]$ and the references therein.

Definition 1.1. A matrix $\mathcal{H} \in \mathbf{C}^{2 n, 2 n}$ is called Hamiltonian if $J_{n} \mathcal{H}=\left(J_{n} \mathcal{H}\right)^{H}$ is Hermitian, where $J_{n}=\left[\begin{array}{cc}0 & I_{n} \\ -I_{n} & 0\end{array}\right], I_{n}$ is the $n \times n$ identity matrix and the superscript $H$ denotes the conjugate transpose.

Every Hamiltonian matrix $\mathcal{H}$ has the block form

$$
\mathcal{H}=\left[\begin{array}{cc}
A & M \\
G & -A^{H}
\end{array}\right]
$$

with $M=M^{H}, G=G^{H}$. Hamiltonian matrices are closely related to algebraic Riccati equations of the form

$$
A^{H} X+X A-X M X+G=0 .
$$

It is well known [15] that if $X=X^{H}$ solves (1.1), then

$$
\mathcal{H}\left[\begin{array}{cc}
I_{n} & 0 \\
-X & I_{n}
\end{array}\right]=\left[\begin{array}{cc}
I_{n} & 0 \\
-X & I_{n}
\end{array}\right]\left[\begin{array}{cc}
(A-M X) & M \\
0 & -(A-M X)^{H}
\end{array}\right] .
$$

This implies that the columns of $\left[\begin{array}{c}I_{n} \\ -X\end{array}\right]$ span an invariant subspace of $\mathcal{H}$ associated with the eigenvalues of $A-M X$. Invariant subspaces of this form are called graph subspaces [15]. The graph subspaces of Hamiltonian matrices are special Lagrangian subspaces.

Definition 1.2. A subspace $\mathbf{L}$ of $\mathbf{C}^{2 n}$ is called Lagrangian subspace if it has dimension $n$ and

$$
x^{H} J_{n} y=0, \quad \forall x, y \in \mathbf{L} .
$$

Clearly a subspace $\mathbf{L}$ is Lagrangian if and only if every matrix $L$ whose columns span $\mathbf{L}$ satisfies $\operatorname{rank} L=n$ and $L^{H} J_{n} L=0$.

\footnotetext{
${ }^{*}$ Fachbereich Mathematik, Universität Duisburg, D-47048 Duisburg, FR Germany

$\dagger$ Institut für Mathematik, MA 4-5, TU Berlin, Str. des 17. Juni 136, D-10623 Berlin, FR Germany. This work was supported by Deutsche Forschungsgemeinschaft, Research Grant Me 790/72 .

$\ddagger$ Department of Mathematics, University of Kansas, Lawrence, Kansas 66045, USA. This work was supported by Deutsche Forschungsgemeinschaft, Research Grant Me 790/7-2.
} 
Despite the fact that Hamiltonian matrices, algebraic Riccati equations and their properties have been a very active area of research for the last 40 years, there are still many open problems. These problems are mainly concerned with Hamiltonian matrices that have eigenvalues with zero real part and in particular with numerical methods for such problems.

In this paper we summarize and extend the known conditions for existence of Lagrangian invariant subspaces of a Hamiltonian matrix. Based on these results we then give a complete parametrization of all possible Lagrangian invariant subspaces and also discuss necessary and sufficient conditions for the uniqueness of Lagrangian invariant subspaces.

Most of the literature on this topic is stated in terms of Hermitian solutions for algebraic Riccati equations, see [15]. For several reasons we will, however, be mainly concerned with the characterization of Lagrangian invariant subspaces. First of all, the concept of Lagrangian invariant subspaces is a more general concept than that of Hermitian solutions of the Riccati equation, since only graph subspaces are associated with Riccati solutions. A second and more important reason is that in most applications the solution of the Riccati equation is not the primary goal, but rather a dangerous detour, see [21]. Finally, even most numerical solution methods for the solution of the algebraic Riccati equations (with the exception of Newton's method) proceed via the computation of Lagrangian invariant subspaces to determine the solution of the Riccati equation, see [3, 5, 6, 7, 8, 16, 17, 20, 27]. These methods employ transformations with symplectic matrices.

Definition 1.3. A matrix $\mathcal{S} \in \mathbf{C}^{2 n, 2 n}$ is called symplectic if $\mathcal{S}^{H} J_{n} \mathcal{S}=J_{n}$. If $\mathcal{S}$ is symplectic then by definition its first $n$ columns span a Lagrangian subspace. Conversely, if the columns of $S_{1}$ span a Lagrangian subspace, then it generates a symplectic matrix, given for example by $\mathcal{S}=\left[S_{1}, J_{n} S_{1}\left(S_{1}^{H} S_{1}\right)^{-1}\right]$. Hence the relation between Lagrangian subspaces and symplectic matrices can be summarized has follows.

Proposition 1.4. If $\mathcal{S} \in \mathbf{C}^{2 n, 2 n}$ is symplectic, then the columns of $\mathcal{S}\left[\begin{array}{c}I_{n} \\ 0\end{array}\right]$ span a Lagrangian subspace. If the columns of $S_{1} \in \mathbf{C}^{2 n, n}$ span a Lagrangian subspace, then there exists a symplectic $\mathcal{S}$ such that range $\mathcal{S}\left[\begin{array}{c}I_{n} \\ 0\end{array}\right]=\operatorname{range} S_{1}$.

Considering Lagrangian invariant subspaces $\mathbf{L}$ of a Hamiltonian matrix $\mathcal{H}$ we immediately have the following important equivalence.

Proposition 1.5. Let $\mathcal{H} \in \mathbf{C}^{2 n, 2 n}$ be a Hamiltonian matrix. There exists a Lagrangian invariant subspace $\mathbf{L}$ of $\mathcal{H}$ if and only if there exists a symplectic matrix $\mathcal{S}$ such that range $\mathcal{S}\left[\begin{array}{c}I_{n} \\ 0\end{array}\right]=\mathbf{L}$ and

$$
\mathcal{S}^{-1} \mathcal{H S}=\left[\begin{array}{cc}
R & D \\
0 & -R^{H}
\end{array}\right]
$$

The form (1.3) is called Hamiltonian block triangular form and if furthermore $R$ is upper triangular (or quasi upper triangular in the real case), it is called Hamiltonian triangular form or Hamiltonian Schur form. Note that for the existence of Lagrangian invariant subspaces it is not necessary that $R$ in (1.3) is triangular if one is not interested in displaying actual eigenvalues. Most numerical methods, however, will return a Hamiltonian triangular or quasi-triangular form.

Necessary and sufficient conditions for the existence of such transformations were given in [18, 22] and in full generality in [19], and we will briefly summarize these 
conditions in the next section. Numerically backward stable methods to compute such forms have been developed in $[1,2,3,4]$.

The contents of this paper is as follows. In Section 2 after recalling some of the results on Hamiltonian triangular forms we will discuss the existence of Lagrangian invariant subspaces corresponding to all possible eigenvalue selections. In Section 3 we give complete parametrizations of all possible Lagrangian subspaces of a Hamiltonian matrix associated with a particular set of eigenvalues. Based on these results we summarize necessary and sufficient conditions for the existence and uniqueness of Lagrangian invariant subspaces in Section 4. Finally we apply these results to give some simple proofs of (mostly known) theorems on existence and uniqueness of Hermitian solutions to algebraic Riccati equations in Section 5.

2. Hamiltonian block triangular forms and existence of Lagrangian invariant subspaces. To study an invariant subspace problem we first need to discuss the possible selection of associated eigenvalues.

We denote by $\Lambda(A)$ the spectrum of a square matrix $A$, counting multiplicities. For a Hamiltonian matrix, if $\lambda \in \Lambda(\mathcal{H})$ and $\operatorname{Re} \lambda \neq 0$ then it is easy to see that also $-\bar{\lambda} \in \Lambda(\mathcal{H})$, see $[15,20]$. Furthermore, if $\mathcal{H}$ has the block triangular form (1.3) and if $i \alpha$ is a purely imaginary eigenvalue (including zero), then it must have even algebraic multiplicity. It follows that the spectrum of a Hamiltonian matrix $\mathcal{H}$ in the form (1.3) can be partitioned into two disjoint subsets

$$
\begin{aligned}
& \Lambda_{1}(\mathcal{H})=\{\underbrace{\lambda_{1}, \ldots, \lambda_{1}}_{n_{1}}, \underbrace{-\bar{\lambda}_{1}, \ldots,-\bar{\lambda}_{1}}_{n_{1}}, \ldots, \underbrace{\lambda_{\mu}, \ldots, \lambda_{\mu}}_{n_{\mu}}, \underbrace{-\bar{\lambda}_{\mu}, \ldots,-\bar{\lambda}_{\mu}}_{n_{\mu}}\} \\
& \Lambda_{2}(\mathcal{H})=\{\underbrace{i \alpha_{1}, \ldots, i \alpha_{1}}_{2 m_{1}}, \ldots, \underbrace{i \alpha_{\nu}, \ldots, i \alpha_{\nu}}_{2 m_{\nu}}\},
\end{aligned}
$$

where $\lambda_{1}, \ldots, \lambda_{\mu}$ are pairwise disjoint eigenvalues with positive real part and $i \alpha_{1}, \ldots$, $i \alpha_{\nu}$ are pairwise disjoint purely imaginary eigenvalues (including zero).

If a matrix is transformed as in (1.3), then the spectrum associated with the Lagrangian invariant subspace spanned by the first $n$ columns of $\mathcal{S}$ is $\Lambda(R)$. Since $\Lambda(\mathcal{H})=\Lambda(R) \cup \Lambda\left(-R^{H}\right)$, it follows that $\Lambda(R)$ must be associated to a characteristic polynomial

$$
\prod_{j=1}^{\mu}\left(\lambda-\lambda_{j}\right)^{t_{j}}\left(\lambda+\bar{\lambda}_{j}\right)^{n_{j}-t_{j}} \prod_{j=1}^{\nu}\left(\lambda-i \alpha_{j}\right)^{m_{j}},
$$

where $t_{j}$ are integers with $0 \leq t_{j} \leq n_{j}$ for $j=1, \ldots, \mu$. We denote the set of all possible such selections of eigenvalues by $\Omega(\mathcal{H})$. Note that $\Omega(\mathcal{H})$ contains $\prod_{j=1}^{\mu}\left(n_{j}+1\right)$ different selections.

In most applications it is desired to determine Lagrangian invariant subspaces associated with eigenvalue selections for which only one of the eigenvalues of the pair $\lambda_{j},-\bar{\lambda}_{j}$ (which are not purely imaginary) can be chosen in $\Lambda(\mathcal{R})$. In another words, $t_{j}$ must be either 0 or $n_{j}$. Such subspaces all called unmixed and the associated Riccati solution, if it exists, is called unmixed solution of the Riccati equation, see [26]. We denote the subset of all possible such selections by $\tilde{\Omega}(\mathcal{H})$. Obviously $\tilde{\Omega}(\mathcal{H})$ contains $2^{\mu}$ different elements.

Note that all selections in $\Omega(\mathcal{H})$ contain the same purely imaginary eigenvalues. Note further that if $\mathcal{H}$ cannot be transformed to the Hamiltonian block triangular form (1.3), then the set $\Omega(\mathcal{H})$ may be empty. A simple example for this is the matrix $J_{1}$. 
We now recall some results on the existence of Hamiltonian triangular forms. In the following we denote a single Jordan block associated with an eigenvalue $\lambda$ by $N_{r}(\lambda)=\lambda I_{r}+N_{r}$, where $N_{r}$ is a nilpotent Jordan block of size $r$. We also frequently use the antidiagonal matrices

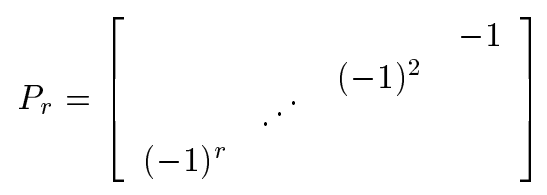

and we denote by $e_{j}$ the $j$-th unit vector of appropriate size.

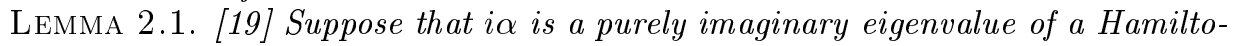
nian matrix $\mathcal{H}$ and that the Jordan block structure associated with this eigenvalue is $N(i \alpha):=i \alpha I+N$, where

$$
N=\operatorname{diag}\left(N_{r_{1}}, \ldots, N_{r_{s}}\right) .
$$

Then there exists a full column rank matrix $U$ such that $\mathcal{H} U=U N(i \alpha)$ and

$$
U^{H} J_{n} U=\operatorname{diag}\left(\pi_{1} P_{r_{1}}, \ldots, \pi_{s} P_{r_{s}}\right),
$$

where $\pi_{k} \in\{1,-1\}$ if $r_{k}$ is even and $\pi_{k} \in\{i,-i\}$ if $r_{k}$ is odd.

Using the indices and matrices introduced in Lemma 2.1, the structure inertia index associated with the eigenvalue $i \alpha$ is defined as

$$
\operatorname{Ind}_{S}(i \alpha)=\left\{\beta_{1}, \ldots, \beta_{s}\right\},
$$

where $\beta_{k}=(-1)^{\frac{r_{k}}{2}} \pi_{k}$ if $r_{k}$ is even, and $\beta_{k}=(-1)^{\frac{r_{k}-1}{2}} i \pi_{k}$ if $r_{k}$ is odd. Note that the $\beta_{i}$ are all \pm 1 and there is one index associated with every Jordan block. The structure inertia index is closely related to the well-known sign characteristic for Hermitian pencils, see [15], since every Hamiltonian matrix $\mathcal{H}$ can be associated with the Hermitian pencil $\lambda i J-J \mathcal{H}$. Although the sign characteristic is a more general concept, since it also applies to general Hermitian pencils, we prefer to use the structure inertia index, since it is better suited for the analysis of Hamiltonian triangular forms, see [19].

For the following analysis the tuple $\operatorname{Ind}_{S}(i \alpha)$ is partitioned into three parts $\operatorname{Ind}_{S}^{e}(i \alpha), \operatorname{Ind}_{S}^{c}(i \alpha), \operatorname{Ind}_{S}^{d}(i \alpha)$, where $\operatorname{Ind}_{S}^{e}(i \alpha)$ contains all the structure inertia indices corresponding to even $r_{k}$, $\operatorname{Ind}_{S}^{c}(i \alpha)$ contains the maximal number of structure inertia indices corresponding to odd $r_{k}$ in \pm 1 pairs and $\operatorname{Ind}_{S}^{d}(i \alpha)$ contains the remaining indices, i.e., all indices in $\operatorname{Ind}_{S}^{d}(i \alpha)$ have the same sign, see [19].

Necessary and sufficient conditions for the existence of a symplectic similarity transformation to a Hamiltonian triangular Jordan like form (1.3) are given in the following Theorem.

TheOREM 2.2. Let $\mathcal{H}$ be a Hamiltonian matrix, let $i \alpha_{1}, \ldots, i \alpha_{\nu}$ be its pairwise distinct purely imaginary eigenvalues and let the columns of $U_{k}, k=1, \ldots, \nu$, span the associated invariant subspaces of dimension $m_{k}$. Then the following are equivalent.

i) There exists a symplectic matrix $\mathcal{S}$, such that $\mathcal{S}^{-1} \mathcal{H S}$ is Hamiltonian block triangular.

ii) There exists a unitary symplectic matrix $\mathcal{U}$, such that $\mathcal{U}^{H} \mathcal{H} \mathcal{U}$ is Hamiltonian block triangular.

iii) $U_{k}^{H} J U_{k}$ is congruent to $J_{m_{k}}$ for all $k=1, \ldots, \nu$.

iv) $\operatorname{Ind}_{S}^{d}\left(i \alpha_{k}\right)$ is void for all $k=1, \ldots, \nu$. 
Moreover, if any of the equivalent conditions holds, then the symplectic matrix $\mathcal{S}$ can be chosen such that $\mathcal{S}^{-1} \mathcal{H S}$ is in Hamiltonian triangular Jordan form

$$
\left[\begin{array}{cccccc}
R_{r} & 0 & 0 & 0 & 0 & 0 \\
0 & R_{e} & 0 & 0 & D_{e} & 0 \\
0 & 0 & R_{c} & 0 & 0 & D_{c} \\
0 & 0 & 0 & -R_{r}^{H} & 0 & 0 \\
0 & 0 & 0 & 0 & -R_{e}^{H} & 0 \\
0 & 0 & 0 & 0 & 0 & -R_{c}^{H}
\end{array}\right]
$$

where the blocks with subscript $r$ are associated with eigenvalues of nonzero real part and have the substructure

$$
R_{r}=\operatorname{diag}\left(R_{1}^{r}, \ldots, R_{\mu}^{r}\right), \quad R_{k}^{r}=\operatorname{diag}\left(N_{d_{k, 1}}\left(\lambda_{k}\right), \ldots, N_{d_{k, p_{k}}}\left(\lambda_{k}\right)\right), \quad k=1, \ldots, \mu .
$$

The blocks with subscript e are associated with the structure inertia indices of even $r_{k}$ for all purely imaginary eigenvalues and have the substructure

$$
\begin{aligned}
& R_{e}=\operatorname{diag}\left(R_{1}^{e}, \ldots, R_{\nu}^{e}\right), \quad R_{k}^{e}=\operatorname{diag}\left(N_{l_{k, 1}}\left(i \alpha_{k}\right), \ldots, N_{l_{k, q_{k}}}\left(i \alpha_{k}\right)\right), \\
& D_{e}=\operatorname{diag}\left(D_{1}^{e}, \ldots, D_{\nu}^{e}\right), \quad D_{k}^{e}=\operatorname{diag}\left(\beta_{k, 1}^{e} e_{l_{k, 1}} e_{l_{k, 1}}^{H}, \ldots, \beta_{k, q_{k}}^{e} e_{l_{k, q_{k}}} e_{l_{k, q_{k}}}^{H}\right) .
\end{aligned}
$$

The blocks with subscript c are associated with pairs of blocks of inertia indices associated with odd-sized blocks for purely imaginary eigenvalues and have the substructure

$$
\begin{aligned}
& R_{c}=\operatorname{diag}\left(R_{1}^{c}, \ldots, R_{\nu}^{c}\right), \quad R_{k}^{c}=\operatorname{diag}\left(B_{k, 1}, \ldots, B_{k, r_{k}}\right), \\
& D_{c}=\operatorname{diag}\left(D_{1}^{c}, \ldots, D_{\nu}^{c}\right), \quad D_{k}^{c}=\operatorname{diag}\left(C_{k, 1}, \ldots, C_{k, r_{k}}\right),
\end{aligned}
$$

where

$$
\begin{aligned}
B_{k, j} & =\left[\begin{array}{ccc}
N_{m_{k, j}}\left(i \alpha_{k}\right) & 0 & -\frac{\sqrt{2}}{2} e_{m_{k, j}} \\
0 & N_{n_{k, j}}\left(i \alpha_{k}\right) & -\frac{\sqrt{2}}{2} e_{n_{k, j}} \\
0 & 0 & i \alpha_{k}
\end{array}\right], \\
C_{k, j} & =\frac{\sqrt{2}}{2} i \beta_{k, j}^{c}\left[\begin{array}{ccc}
0 & 0 & e_{m_{k, j}} \\
0 & 0 & -e_{n_{k, j}} \\
-e_{m_{k, j}}^{H} & e_{n_{k, j}}^{H} & 0
\end{array}\right] .
\end{aligned}
$$

Proof. The proof of equivalence for i) and iv) is given in Theorem 1.3 in [25]. The equivalence of the other conditions and the structured Hamiltonian triangular Jordan form (2.3) has been derived in [19].

REMARK 1. For real Hamiltonian matrices a real quasi triangular Jordan form analogous to (2.3) and a similar set of equivalent conditions as in Theorem 2.2 can be given. We refer the reader to [25] and Theorem 24 in [19] for details. The necessary and sufficient conditions in Theorem 2.2 only guarantee the existence of one Lagrangian invariant subspace associated to one selection in $\Omega(\mathcal{H})$. But the following theorem shows they also guarantee the existence of a Lagrangian invariant subspace associated to every selection in $\Omega(\mathcal{H})$.

THEOREM 2.3. Let $\mathcal{H}$ be a Hamiltonian matrix. If any of the conditions in Theorem 2.2 holds, then for every eigenvalue selection $\omega \in \Omega(\mathcal{H})$ there exists at least one corresponding Lagrangian invariant subspace.

Proof. A proof for this result based on condition iv) was given in [23, 25] but a simple proof follows directly from (2.3). Note that any $\omega$ contains half the number 
of eigenvalues for every purely imaginary eigenvalue. So a basis for a corresponding invariant subspace is easily determined from (2.3). For an eigenvalue pair $\lambda_{k},-\bar{\lambda}_{k}$ we only need to consider the small Hamiltonian block $\left[\begin{array}{cc}R_{k}^{r} & 0 \\ 0 & -\left(R_{k}^{r}\right)^{H}\end{array}\right]$. Note that $R_{k}^{r}$ is upper triangular. Suppose that the selection $\omega$ contains $t_{k}$ copies of $\lambda_{k}$ and $s_{k}$ copies of $-\bar{\lambda}_{k}$. A corresponding basis of the invariant subspace can then be chosen based on a symplectic permutation which exchanges trailing $s_{k} \times s_{k}$ blocks in $R_{k}^{r}$ and $-\left(R_{k}^{r}\right)^{H}$.

(

In this section we have reviewed some results on the existence of (unitary) symplectic transformations to Hamiltonian block triangular form, and the existence of Lagrangian invariant subspaces. In the next section we use these results to give a full parametrization of all possible Lagrangian subspaces and therefore also a parametrization of all symplectic similarity transformations to Hamiltonian block triangular form.

3. Parametrization of all Lagrangian invariant subspaces. In the previous section we have shown that if $\mathcal{H}$ has a Hamiltonian block triangular form then for every eigenvalue selection $\omega \in \Omega(\mathcal{H})$ there exists at least one corresponding invariant subspace. In this section we will parametrize all possible Lagrangian invariant subspaces associated to a given selection $\omega$.

For this we will need some technical lemmas.

Lemma 3.1. Consider pairs of matrices $\left(\pi_{k} P_{r_{k}}, N_{r_{k}}\right), k=1,2$, where $r_{1}, r_{2}$ are either both even or both odd. Let $\pi_{1}, \pi_{2} \in\{1,-1\}$ if both $r_{k}$ are even and $\pi_{1}, \pi_{2} \in$ $\{i,-i\}$ if both $r_{k}$ are odd, let

$$
\left(\mathcal{P}_{c}, \mathcal{N}_{c}\right):=\left(\left[\begin{array}{cc}
\pi_{1} P_{r_{1}} & 0 \\
0 & \pi_{2} P_{r_{2}}
\end{array}\right],\left[\begin{array}{cc}
N_{r_{1}} & 0 \\
0 & N_{r_{2}}
\end{array}\right]\right)
$$

and let $d:=\frac{\left|r_{1}-r_{2}\right|}{2}$. If $\pi_{1}=(-1)^{d+1} \pi_{2}$, i.e., $\beta_{1}=-\beta_{2}$ for the corresponding $\beta_{1}$ and $\beta_{2}$, then we have the following transformations to Hamiltonian triangular form.

1. If $r_{1} \geq r_{2}$ then with

$$
Z_{1}:=\left[\begin{array}{cccc}
I_{d} & 0 & 0 & 0 \\
0 & I_{r_{2}} & 0 & -\frac{1}{2} \bar{\pi}_{2} P_{r_{2}}^{-1} \\
0 & 0 & \bar{\pi}_{1} P_{d}^{-1} & 0 \\
0 & -I_{r_{2}} & 0 & -\frac{1}{2} \bar{\pi}_{2} P_{r_{2}}^{-1}
\end{array}\right]
$$

we obtain $Z_{1}^{H} \mathcal{P}_{c} Z_{1}=J_{\frac{r_{1}+r_{2}}{2}}$ and

$$
Z_{1}^{-1} \mathcal{N}_{c} Z_{1}=\left[\begin{array}{cc}
N_{\frac{r_{1}+r_{2}}{2}} & D \\
0 & -N_{\frac{r_{1}+r_{2}}{2}}^{H}
\end{array}\right],
$$

where $D=\tau e_{d} e_{\frac{r_{1}+r_{2}}{2}}^{H}+\bar{\tau} e_{\frac{r_{1}+r_{2}}{2}} e_{d}^{H}, \tau=-\frac{1}{2} \pi_{2}$.

2. If $r_{1}<r_{2}$, then with

$$
Z_{2}=\left[\begin{array}{cccc}
\pi_{1} P_{r_{1}} & 0 & \frac{1}{2} I_{r_{1}} & 0 \\
0 & \pi_{2} P_{d} & 0 & 0 \\
-\pi_{1} P_{r_{1}} & 0 & \frac{1}{2} I_{r_{1}} & 0 \\
0 & 0 & 0 & I_{d}
\end{array}\right]
$$

we obtain that $Z_{2}^{H} \mathcal{P}_{c} Z_{2}=J_{\frac{r_{1}+r_{2}}{2}}$ and

$$
Z_{2}^{-1} \mathcal{N}_{c} Z_{2}=\left[\begin{array}{cc}
-N_{\frac{r_{1}+r_{2}}{2}}^{H} & D \\
0 & N_{\frac{r_{1}+r_{2}}{2}}^{H}
\end{array}\right],
$$


where $D=\tau e_{1} e_{r_{1}+1}^{H}+\bar{\tau}_{1} e_{r_{1}+1} e_{1}^{H}, \tau=-\frac{1}{2} \pi_{1}$.

Proof. The proof is a simple modification of the proof of Lemma 18 in [19].

Lemma 3.2. Consider a nilpotent matrix in Jordan form $N=\operatorname{diag}\left(N_{r_{1}}, \ldots, N_{r_{p}}\right)$.

i) If the columns of the full column rank matrix $X$ form an invariant subspace of $N$, i.e., $N X=X A$ for some matrix $A$, then $X=U Z$, where $Z$ is nonsingular and

$$
U=\left[\begin{array}{ccccc}
I_{t_{1}} & 0 & \ldots & 0 & 0 \\
0 & V_{1,2} & \ldots & V_{1, p-1} & V_{1, p} \\
0 & I_{t_{2}} & \ldots & 0 & 0 \\
0 & 0 & \ldots & V_{2, p-1} & V_{2 p} \\
\vdots & \vdots & \ddots & \vdots & \vdots \\
0 & 0 & \ldots & I_{t_{p-1}} & 0 \\
0 & 0 & \ldots & 0 & V_{p-1, p} \\
0 & 0 & \ldots & 0 & I_{t_{p}} \\
0 & 0 & \cdots & 0 & 0
\end{array}\right]
$$

Here for $k=1, \ldots, p, 0 \leq t_{k} \leq r_{k}$, and for $i=1, \ldots, p-1$ and $j=i+1, \ldots, p$, we have $V_{i, j} \in \mathbf{C}^{s_{i}, t_{j}}$ with $s_{i}=r_{i}-t_{i}$. Moreover, if $M_{s}=\operatorname{diag}\left(N_{s_{1}}, \ldots, N_{s_{p}}\right)$, $M_{t}=\operatorname{diag}\left(N_{t_{1}}, \ldots, N_{t_{p}}\right)$ and $E=\operatorname{diag}\left(e_{t_{1}} e_{1}^{H}, \ldots, e_{t_{p}} e_{1}^{H}\right)$, then

$$
V=\left[\begin{array}{cccc}
0 & V_{12} & \cdots & V_{1 p} \\
& \ddots & \ddots & \vdots \\
& & \ddots & V_{p-1, p} \\
& & & 0
\end{array}\right]
$$

satisfies the algebraic Riccati equation

$$
M_{s} V-V M_{t}-V E V=0 .
$$

ii) If the columns of the full column rank matrix $X$ form an invariant subspace of $N^{H}$, i.e., $N^{H} X=-X A$ for some matrix $A$, then $X=\hat{U} Z$, where $Z$ is nonsingular and

$$
\hat{U}=\left[\begin{array}{ccccc}
0 & 0 & \ldots & 0 & 0 \\
-I_{\hat{t}_{1}} & 0 & \ldots & & 0 \\
\hat{V}_{2,1} & 0 & \ldots & 0 & 0 \\
0 & -I_{\hat{t}_{2}} & \cdots & 0 & 0 \\
\vdots & \vdots & \ddots & \vdots & \vdots \\
\hat{V}_{p-1,1} & \hat{V}_{p-1,2} & \ldots & 0 & 0 \\
0 & 0 & \ldots & -I_{\hat{t}_{p-1}} & 0 \\
\hat{V}_{p, 1} & \hat{V}_{p, 2} & \ldots & \hat{V}_{p, p-1} & 0 \\
0 & 0 & \ldots & 0 & -I_{\hat{t}_{p}}
\end{array}\right] .
$$

Here for $k=1, \ldots, p, 0 \leq \hat{t}_{k} \leq r_{k}$ and for $i=2, \ldots, p$ and $j=1, \ldots, i-1$, we have $\hat{V}_{i, j} \in \mathbf{C}^{\hat{s}_{i}, \hat{t}_{j}}$ with $\hat{s}_{i}=r_{i}-\hat{t}_{i}$. Moreover, if $M_{\hat{s}}=\operatorname{diag}\left(N_{\hat{s}_{1}}, \ldots, N_{\hat{s}_{p}}\right)$, 
$M_{\hat{t}}=\operatorname{diag}\left(N_{\hat{t}_{1}}, \ldots, N_{\hat{t}_{p}}\right)$ and $E=\operatorname{diag}\left(e_{1} e_{\hat{s}_{1}}^{H}, \ldots, e_{1} e_{\hat{s}_{p}}^{H}\right)$, then

$$
\hat{V}=\left[\begin{array}{cccc}
0 & & & \\
\hat{V}_{21} & \ddots & & \\
\vdots & \ddots & \ddots & \\
\hat{V}_{p 1} & \ldots & \hat{V}_{p, p-1} & 0
\end{array}\right]
$$

satisfies the algebraic Riccati equation

$$
M_{\hat{s}}^{H} \hat{V}-\hat{V} M_{\hat{t}}^{H}-\hat{V} E \hat{V}=0 .
$$

Proof. We will derive the structure of $X$ by multiplying nonsingular matrices to $X$ from the right. Let us first prove part i). Partition $X=\left[\begin{array}{l}X_{1} \\ X_{2}\end{array}\right]$ so that $X_{2}$ has $r_{p}$ rows. Then using the $\mathrm{QR}$ or singular value decomposition [12], there exists a nonsingular (actually unitary) matrix $Y_{1}$ such that $X_{2}=\left[0, X_{22}\right] Y_{1}$, where $X_{22} \in$ $\mathbf{C}^{r_{p}, t_{p}}$ and $\operatorname{rank} X_{22}=t_{p}$. (This implies that $0 \leq t_{p} \leq r_{p}$.) Then we have the partition $\hat{X}=X Y_{1}^{-1}=\left[\begin{array}{cc}X_{11} & X_{12} \\ 0 & X_{22}\end{array}\right]$. Since range $X$ is an invariant subspace of $N$ so is range $\hat{X}$. Hence, there exists a matrix $\hat{A}$ such that

$$
N \hat{X}=\hat{X} \hat{A}
$$

Partitioning $\hat{A}=\left[\begin{array}{ll}A_{11} & A_{12} \\ A_{21} & A_{22}\end{array}\right]$ conformally with $\hat{X}$, then (3.3) implies that $A_{21}=0$ and $N_{r_{p}} X_{22}=X_{22} A_{22}$. Because $X_{22}$ has full column rank and $N_{r_{p}}$ is a single Jordan block, it is clear that $A_{22}$ is similar to $N_{t_{p}}$, i.e., there exists a nonsingular matrix $Y_{22}$ such that $Y_{22}^{-1} A_{22} Y_{22}=N_{t_{p}}$ and hence $N_{r_{p}}\left(X_{22} Y_{22}\right)=\left(X_{22} Y_{22}\right) N_{t_{p}}$. By Lemma 4.4.11 in [14], $X_{22} Y_{22}=\left[\begin{array}{c}T \\ 0\end{array}\right]$, where $T$ is an upper triangular Toeplitz matrix and $T$ must be nonsingular, since $X_{22}$ has full column rank. Therefore, by setting $\tilde{X}=\hat{X} Y_{2}$ with $Y_{2}=\operatorname{diag}\left(I, Y_{22}\right) T^{-1}$, it follows that

$$
\tilde{X}=\left[\begin{array}{cc}
\tilde{X}_{1} & \tilde{X}_{2} \\
0 & I_{t_{p}} \\
0 & 0
\end{array}\right]
$$

and (3.3) becomes $N \tilde{X}=\tilde{X}\left[\begin{array}{cc}\tilde{A}_{11} & \tilde{A}_{12} \\ 0 & N_{t_{p}}\end{array}\right]$. Setting $\tilde{N}=\operatorname{diag}\left(N_{r_{1}}, \ldots, N_{r_{p-1}}\right)$ it follows that $\tilde{N} \tilde{X}_{1}=\tilde{X}_{1} \tilde{A}_{11}$, and since $X$ has full column rank, also $\tilde{X}_{1}$ has full column rank.

By inductively applying the construction that leads from $X$ to $\tilde{X}$, we determine a nonsingular matrix $Z_{1}$ such that $X Z_{1}^{-1}=\breve{X}$, where $\breve{X}$ has the block structure

$$
\breve{X}=\left[\begin{array}{ccccc}
I_{t_{1}} & W_{1,2} & \ldots & W_{1, p-1} & W_{1, p} \\
0 & V_{1,2} & \ldots & V_{1, p-1} & V_{1, p} \\
0 & I_{t_{2}} & \ldots & W_{2, p-1} & W_{2, p} \\
0 & 0 & \ldots & V_{2, p-1} & V_{2, p} \\
\vdots & \vdots & \ddots & \vdots & \vdots \\
0 & 0 & \ldots & I_{t_{p-1}} & W_{p-1, p} \\
0 & 0 & \ldots & 0 & V_{p-1, p} \\
0 & 0 & \ldots & 0 & I_{t_{p}} \\
0 & 0 & \ldots & 0 & 0
\end{array}\right],
$$


with $0 \leq t_{i} \leq r_{i}$. The blocks $W_{i, j}$ in $\breve{X}$ can be eliminated by performing a sequence of block Gaussian type eliminations from the right. Hence, there exists a nonsingular matrix $Z_{2}$ such that $\breve{X} Z_{2}^{-1}=U$, where $U$ is in (3.1). Therefore, by setting $Z:=Z_{2} Z_{1}$ we have $X=U Z$.

From the block form of $U$ we can determine a block permutation matrix $Q$ such that $Q U=\left[\begin{array}{c}I \\ V\end{array}\right]$ and $Q N Q^{-1}=\left[\begin{array}{cc}M_{t} & E \\ 0 & M_{s}\end{array}\right]$. Since $\left[\begin{array}{l}I \\ V\end{array}\right]$ is invariant to $Q N Q^{-1}$, we have $M_{s} V-V M_{t}-V E V=0$.

Part (ii) is proved analogously by beginning the reduction from the top and compressing in each step to the left.

Using these lemmas we are able to parametrize the set of all Lagrangian invariant subspaces of a Hamiltonian matrix $\mathcal{H}$ associated with a fixed eigenvalue selection in $\omega \in \Omega(\mathcal{H})$. Let $\mathcal{H}$ be in Hamiltonian block triangular form (1.3) and let the spectrum of $\mathcal{H}$ be as in (2.1). Then, see [19], there exists a symplectic matrix $\mathcal{S}$ such that $\mathcal{S}^{-1} \mathcal{H S}=\left[\begin{array}{cc}R & D \\ 0 & -R^{H}\end{array}\right]$, where $R=\operatorname{diag}\left(R_{1}, \ldots, R_{\mu+\nu}\right)$ and $D=\operatorname{diag}\left(D_{1}, \ldots, D_{\mu+\nu}\right)$. Furthermore, the blocks are reordered such that $H_{k}:=\left[\begin{array}{cc}R_{k} & D_{k} \\ 0 & -R_{k}^{H}\end{array}\right]$ is Hamiltonian block triangular, and associated with an eigenvalue pair $\lambda_{k},-\bar{\lambda}_{k}$ with nonzero real part for $k=1, \ldots, \mu$ and purely imaginary eigenvalues $i \alpha_{k}$ for $k=\mu+1, \ldots, \mu+\nu$. Furthermore, $\Lambda(R)=\omega$ and range $\mathcal{S}\left[\begin{array}{l}I \\ 0\end{array}\right]=\mathbf{L}$.

For this block diagonal form there exists a block permutation matrix $\mathcal{P}$ such that

$$
\begin{aligned}
\mathcal{P}^{H} J \mathcal{P} & =\operatorname{diag}\left(J_{n_{1}}, \ldots, J_{n_{\mu}} ; J_{m_{1}}, \ldots, J_{m_{\nu}}\right)=: \tilde{J} \\
\mathcal{P}^{-1} \mathcal{S}^{-1} \mathcal{H S P} & =\operatorname{diag}\left(H_{1}, \ldots, H_{\mu} ; H_{\mu+1}, \ldots, H_{\mu+\nu}\right) .
\end{aligned}
$$

Suppose that there exists another Lagrangian invariant subspace $\tilde{\mathbf{L}}$ corresponding to $\omega$. Using the same argument, there exists a symplectic matrix $\tilde{\mathcal{S}}$, such that for the same block permutation matrix $\mathcal{P}$ we have

$$
\mathcal{P}^{-1} \tilde{\mathcal{S}}^{-1} \mathcal{H} \tilde{\mathcal{S}} \mathcal{P}=\operatorname{diag}\left(\tilde{H}_{1}, \ldots, \tilde{H}_{\mu} ; \tilde{H}_{\mu+1}, \ldots, \tilde{H}_{\mu+\nu}\right),
$$

where again all $\tilde{H}_{k}$ are Hamiltonian block triangular and $\Lambda\left(\tilde{H}_{k}\right)=\Lambda\left(H_{k}\right)$ for all $k=1, \ldots, \mu+\nu$. Therefore, we have $\tilde{\mathcal{S}} \mathcal{P}=\mathcal{S P} \mathcal{E}$, for some block diagonal matrix $\mathcal{E}=\operatorname{diag}\left(E_{1}, \ldots, E_{\mu+\nu}\right)$ satisfying $H_{k} E_{k}=E_{k} \tilde{H}_{k}$. Since $\mathcal{P}^{H} J \mathcal{P}=\tilde{J}$ and since $\mathcal{S}$ and $\tilde{\mathcal{S}}$ are symplectic, it follows that $\mathcal{E}=\mathcal{P}^{-1} \mathcal{S}^{-1} \tilde{\mathcal{S}} \mathcal{P}$ satisfies $\mathcal{E}^{H} \tilde{J} \mathcal{E}=\tilde{J}$, which implies that all blocks $E_{k}$ are symplectic. Since $\tilde{\mathcal{S}}=\mathcal{S P} \mathcal{E} \mathcal{P}^{-1}$, the difference between $\tilde{\mathcal{S}}$ and $\mathcal{S}$, (and therefore $\tilde{\mathbf{L}}$ and $\mathbf{L}$ ) is completely described by the first half of the columns of the symplectic matrices $E_{k}$, i.e., the Lagrangian invariant subspaces of the small Hamiltonian matrices $H_{k}$ (note that all $\tilde{H}$ are Hamiltonian block triangular). Following this argument, it is sufficient to parametrize all possible Lagrangian invariant subspaces of a Hamitonian matrix with either a single purely imaginary eigenvalue $i \alpha$ or a single eigenvalue pair $\lambda,-\bar{\lambda}$ with $\operatorname{Re} \lambda \neq 0$.

Consider first the case of a single purely imaginary eigenvalue. In this case $\Omega(\mathcal{H})$ has only one element. So all Lagrangian invariant subspaces are associated to the same eigenvalue.

To simplify our analysis we need the following Hamiltonian Jordan form.

Lemma 3.3. Let $\mathcal{H}$ be a Hamiltonian matrix that has only one eigenvalue $i \alpha$. Then there exists a symplectic matrix $\mathcal{S}$ such that

$$
\mathcal{R}:=\mathcal{S}^{-1} \mathcal{H S}=\left[\begin{array}{cc}
N(i \alpha) & D \\
0 & -N(i \alpha)^{H}
\end{array}\right],
$$


where $N=\operatorname{diag}\left(N_{r_{1}}, \ldots, N_{r_{p}}\right), D=\operatorname{diag}\left(D_{1}, \ldots, D_{p}\right)$. Here either $D_{j}=\beta_{j}^{e} e_{r_{j}} e_{r_{j}}^{H}$, so that $\mathcal{H}$ has a Jordan block $N_{2 r_{j}}$ with structure inertia index $\beta_{j}^{e} \in\{1,-1\}$, or $D_{j}=$ $\tau_{j} e_{d_{j}} e_{r_{j}}^{H}+\bar{\tau}_{j} e_{r_{j}} e_{d_{j}}^{H}$ with $\tau_{j}=\frac{1}{2}(-1)^{\frac{r_{j}+d_{j}+1}{2}} i \beta_{j}$ if $r_{j}+d_{j}$ is odd, and $\tau_{j}=\frac{1}{2}(-1)^{\frac{r_{j}+d_{j}}{2}} \beta_{j}$ if $r_{j}+d_{j}$ is even for some $\beta_{j} \in\{-1,1\}$, so that $\mathcal{H}$ has two Jordan blocks $N_{r_{j}+d_{j}}$, $N_{r_{j}-d_{j}}$ with structure inertia indices $\beta_{j},-\beta_{j}$, respectively.

Proof. Since $\mathcal{H}-i \alpha I$ is Hamiltonian, we may w.l.o.g. consider the problem with $\alpha=0$, i.e., $\mathcal{H}$ that has only the eigenvalue zero. Since $\mathcal{H}$ has only one multiple eigenvalue the columns of every nonsingular matrix span a corresponding invariant subspace so that condition iii) of Theorem 2.2 holds. The canonical form (3.5) then is obtained in a similar way as for (2.3), see [19]. The only difference is here, that we match all possible pairs of Jordan block with opposite structure inertia indices in such a way that even blocks are matched with even blocks and odd blocks with odd blocks and furthermore the blocks are ordered in decreasing size. Finally we use the technique given in Lemma 3.1.

The complete parameterization is then as follows.

THEOREM 3.4. Let $\mathcal{H}$ be a Hamiltonian matrix that has only one purely imaginary eigenvalue. Let $\mathcal{S}$ be symplectic such that $\mathcal{S}^{-1} \mathcal{H} \mathcal{S}$ is in Hamiltonian canonical form (3.5). Then all possible Lagrangian subspaces can be parametrized by range $\mathcal{S} U$, where

$$
(3.6) U=\left[\begin{array}{ccccccccc}
I_{t_{1}} & 0 & \ldots & 0 & 0 & 0 & \ldots & 0 & 0 \\
0 & V_{12} & \ldots & V_{1, p-1} & V_{1 p} & W_{11} & \ldots & W_{1, p-1} & W_{1 p} \\
0 & I_{t_{2}} & \ldots & 0 & 0 & 0 & \ldots & 0 & 0 \\
0 & 0 & \ldots & V_{2, p-1} & V_{2 p} & W_{12}^{H} & \ldots & W_{2, p-1} & W_{2 p} \\
\vdots & \vdots & \ddots & \vdots & \vdots & \vdots & \ddots & \vdots & \vdots \\
0 & 0 & \ldots & I_{t_{p-1}} & 0 & 0 & \ldots & 0 & 0 \\
0 & 0 & \ldots & 0 & V_{p-1, p} & W_{1, p-1}^{H} & \ldots & W_{p-1, p-1} & W_{p-1, p} \\
0 & 0 & \ldots & 0 & I_{t_{p}} & 0 & \ldots & 0 & 0 \\
0 & 0 & \ldots & 0 & 0 & W_{1 p}^{H} & \ldots & W_{p-1, p}^{H} & W_{p p} \\
0 & 0 & \ldots & 0 & 0 & 0 & \ldots & 0 & 0 \\
0 & 0 & \ldots & 0 & 0 & -I_{s_{1}} & 0 & \ldots & 0 \\
0 & 0 & \ldots & 0 & 0 & V_{12}^{H} & 0 & \ldots & 0 \\
\vdots & \vdots & \ddots & \vdots & \vdots & \vdots & \ddots & \vdots & \vdots \\
0 & 0 & \ldots & 0 & 0 & V_{1, p-1}^{H} & \ldots & 0 & 0 \\
0 & 0 & \ldots & 0 & 0 & 0 & \ldots & -I_{s_{p-1}} & 0 \\
0 & 0 & \ldots & 0 & 0 & V_{1 p}^{H} & \ldots & V_{p-1, p}^{H} & 0 \\
0 & 0 & \ldots & 0 & 0 & 0 & \ldots & 0 & -I_{s_{p}}
\end{array}\right] \text {, }
$$

with block sizes $0 \leq s_{j}, t_{j} \leq r_{j}$ and $s_{j}+t_{k}=r_{j}$. Then, setting

$$
\begin{aligned}
M_{t} & =\operatorname{diag}\left(N_{t_{1}}, \ldots, N_{t_{p}}\right), \\
M_{s} & =\operatorname{diag}\left(N_{s_{1}}, \ldots, N_{s_{p}}\right), \\
E & =\operatorname{diag}\left(e_{t_{1}} e_{1}^{H}, \ldots, e_{t_{p}} e_{1}^{H}\right),
\end{aligned}
$$

partitioning the Hermitian blocks

$$
D_{j}=\left[\begin{array}{cc}
G_{j} & F_{j} \\
F_{j}^{H} & K_{j}
\end{array}\right]
$$

and setting

$$
K=\operatorname{diag}\left(K_{1}, \ldots, K_{s}\right)
$$




$$
\begin{aligned}
& F=\operatorname{diag}\left(F_{1}, \ldots, F_{s}\right), \\
& G=\operatorname{diag}\left(G_{1}, \ldots, G_{s}\right),
\end{aligned}
$$

it follows that the block matrices

$$
V:=\left[\begin{array}{cccc}
0 & V_{1,2} & \ldots & V_{1, p} \\
& \ddots & \ddots & \vdots \\
& & \ddots & V_{p-1, p} \\
& & & 0
\end{array}\right], \quad W:=\left[\begin{array}{ccc}
W_{1,1} & \ldots & W_{1, p} \\
\vdots & \ddots & \vdots \\
W_{1, p}^{H} & \ldots & W_{p, p}
\end{array}\right]=W^{H}
$$

satisfy

$$
\begin{gathered}
{\left[\begin{array}{cc}
M_{s} & F^{H} \\
0 & -M_{t}^{H}
\end{array}\right]\left[\begin{array}{cc}
W & V \\
V^{H} & 0
\end{array}\right]+\left[\begin{array}{cc}
W & V \\
V^{H} & 0
\end{array}\right]\left[\begin{array}{cc}
M_{s}^{H} & 0 \\
F & -M_{t}
\end{array}\right]} \\
-\left[\begin{array}{cc}
W & V \\
V^{H} & 0
\end{array}\right]\left[\begin{array}{cc}
0 & E^{H} \\
E & G
\end{array}\right]\left[\begin{array}{cc}
W & V \\
V^{H} & 0
\end{array}\right]-\left[\begin{array}{cc}
K & 0 \\
0 & 0
\end{array}\right]=0,
\end{gathered}
$$

or equivalently $V, W$ satisfy

$$
\begin{aligned}
0= & M_{s} V-V M_{t}-V E V \\
0= & \left(M_{s}-V E\right) W+W\left(M_{s}-V E\right)^{H} \\
& +(V F)^{H}+V F-V G V^{H}-K .
\end{aligned}
$$

Every Lagrangian invariant subspace is uniquely determined by a set of parameters $t_{1}, \ldots, t_{p}$ with $0 \leq t_{j} \leq r_{j}$, and a set of matrices $V_{i, j}, i=1, \ldots, p-1, j=i+1, \ldots, p$ and $W_{i, j}, i=1, \ldots, p, j=i, \ldots, p$ satisfying (3.8) and (3.9).

Moreover, all symplectic matrices that transform $\mathcal{H}$ to Hamiltonian block triangular form can be parametrized as $\mathcal{S U Y}$, where $\mathcal{Y}$ is a symplectic block triangular matrix, $\mathcal{U}=[U, \tilde{U}]$, with $U$ as in (3.6) and

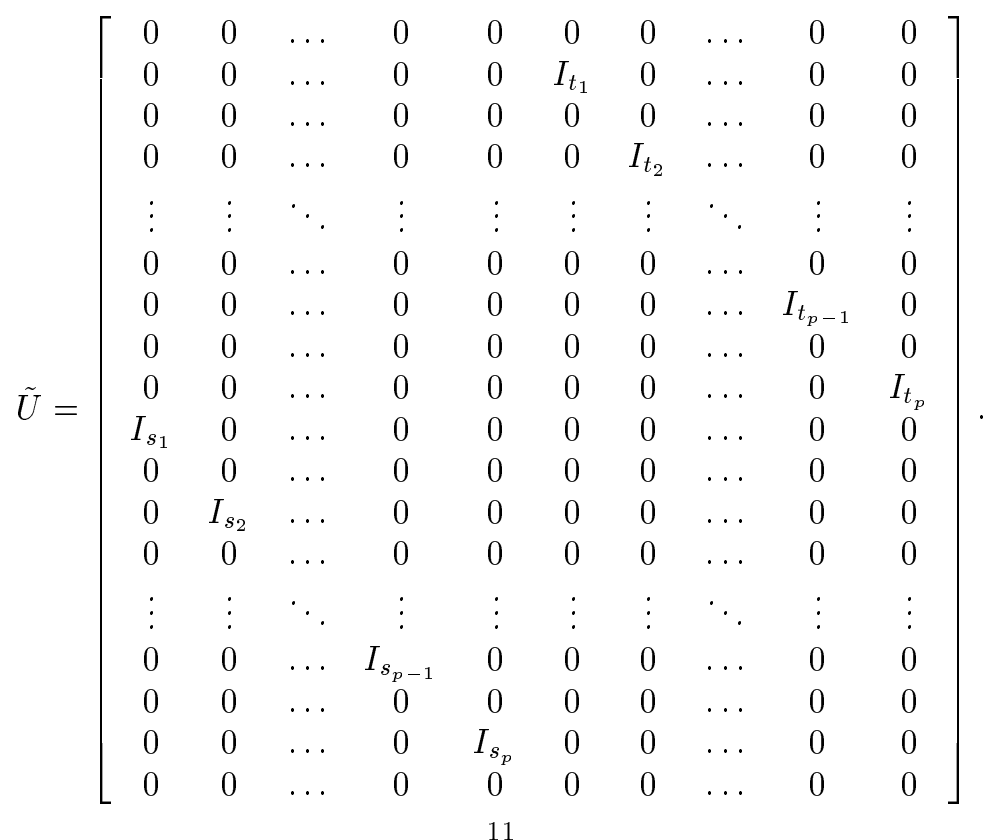


Proof. As in Lemma 3.3, we assume that the only eigenvalue of $\mathcal{H}$ is zero. Considering the form (3.5), it is sufficient to prove that every basis $X$ of a Lagrangian invariant subspace of $\mathcal{R}$ can be expressed as $X=U Y$. To prove this, we first compress the bottom square block of $X$, i.e., we determine a matrix $Y_{1}$ such that $X Y_{1}=\left[\begin{array}{cc}X_{11} & X_{12} \\ 0 & X_{22}\end{array}\right]$ where $X_{22}$ has full column rank. Obviously $X_{11}$ also has full column rank. Then, since $X Y_{1}$ is still a basis of an invariant subspace of $\mathcal{R}$, the block triangular form of $\mathcal{R}$ implies that the columns of $X_{11}$ and $X_{22}$ form bases of the invariant subspace of $N$ and $-N^{H}$, respectively. Applying Lemma 3.2, there exist matrices $Z_{1}$ and $Z_{2}$ such that $U_{11}:=X_{11} Z_{1}$ and $U_{22}:=X_{22} Z_{2}$ have structures as the matrices in (3.1) and (3.2) associated with the integer parameters $t_{1}, \ldots, t_{p}$ and $\hat{t}_{1}, \ldots, \hat{t}_{p}$, respectively. Now let $U:=X Y_{1}\left[\begin{array}{cc}Z_{1} & 0 \\ 0 & Z_{2}\end{array}\right] Y_{2}=\left[\begin{array}{cc}U_{11} & U_{12} \\ 0 & U_{22}\end{array}\right]$, where $Y_{2}$ is used to eliminate the blocks in $X_{12} Z_{2}$ using the identity blocks in $U_{1,1}$. Since $X$ and hence also $U$ is Lagrangian, we have that $U_{11}^{H} U_{22}=0$. Thus, we have $\hat{t}_{j}=m_{j}-t_{j}=: s_{j}$ for all $j=1, \ldots, p$ and $\hat{V}_{i, j}=V_{j, i}^{H}$ for all $i=2, \ldots, p, j=1, \ldots, p-1$. Since $U_{12}^{H} U_{22}$ is Hermitian, it follows that $U_{12}$ has the desired form. To prove (3.7), as in the proof of Lemma 3.2, there exists a block permutation matrix $P$ such that $P\left[U_{11}, U_{12}\right]=\left[\begin{array}{cc}I & 0 \\ V & W\end{array}\right]$. Let $\tilde{\mathcal{P}}=\operatorname{diag}(P, P)$, which is symplectic. Then

$$
\tilde{\mathcal{P}} U=\left[\begin{array}{cc}
I & 0 \\
V & W \\
0 & V^{H} \\
0 & -I
\end{array}\right], \quad \tilde{\mathcal{P}}^{-1} \mathcal{R} \tilde{\mathcal{P}}=\left[\begin{array}{cccc}
M_{t} & E & G & F \\
0 & M_{s} & F^{H} & K \\
0 & 0 & -N_{t}^{H} & 0 \\
0 & 0 & -E^{H} & -N_{s}^{H}
\end{array}\right] .
$$

Since the columns of $\tilde{\mathcal{P}} U$ form an invariant subspace for $\tilde{\mathcal{P}}^{-1} \mathcal{R} \tilde{\mathcal{P}}$, it follows that the matrices $V, W$ satisfy (3.7). Conditions (3.8) and (3.9) follow directly from (3.7). To show the uniqueness of a particular Lagrangian invariant subspace, suppose that there are two matrices $U_{1}, U_{2}$ of the same form as $U$ such that range $\mathcal{S} U_{1}=$ range $\mathcal{S} U_{2}$. Then $U_{2}^{H} J U_{1}=0$ and from this it follows first that the associated integer parameters $t_{1}, \ldots, t_{p}$ must be the same, and then all the blocks $V_{i, j}, W_{i, j}$ must be the same.

To prove the second part, let $\mathcal{X}$ be a symplectic matrix which triangularizes $\mathcal{H}$. Since the first $n$ columns of $\mathcal{X}$ form a Langrangian invariant subspace, there exists a matrix $U$ of the form (3.6) such that range $\mathcal{X}\left[\begin{array}{l}I \\ 0\end{array}\right]=$ range $\mathcal{S} U$. Then the matrix $\mathcal{U}=[U, \tilde{U}]$ with $\tilde{U}$ as in (3.10) is symplectic. Since both $\mathcal{X}$ and $\mathcal{S U}$ are symplectic and their first $n$ columns span the same subspace there exists a symplectic block triangular matrix $\mathcal{Y}$ such that $\mathcal{X}=\mathcal{S U Y}$.

These results show that the parameters that characterize a Lagrangian invariant subspace are integers $t_{j}$ with $0 \leq t_{j} \leq m_{j}$, and the matrices $V_{i, j}, W_{i, j}$ satisfying the Riccati equations (3.7), or equivalently (3.8) and (3.9). Note that the equation for $W$ is a singular Lyapunov equation. The equation for $V$ is quadratic. But if we consider it blockwise, it is equivalent to a sequence of singular Sylvester equations

$$
N_{s_{i}} V_{i, j}-V_{i, j} N_{t_{j}}-\sum_{k=i+1}^{j-1} V_{i, k} E_{k} V_{k, j}=0
$$

for $i=p-1, \ldots, 1, j=i+1, \ldots, p$. For results on nonsymmetric Riccati equations see [10]. 
In general not much more can be said about this parametrization. In the special case of a Hamiltonian matrix $\mathcal{H}$ that has only two Jordan blocks we have the following result.

Corollary 3.5. Consider a Hamiltonian matrix $\mathcal{H}$ that has exactly two Jordan blocks $N_{r_{1}}(i \alpha), N_{r_{2}}(i \alpha)$ with $0<r_{2} \leq r_{1}$ and the corresponding structure inertia indices $\beta_{1}=-\beta_{2}$. Then there exists a symplectic matrix $\mathcal{S}$ such that

$$
\mathcal{S}^{-1} \mathcal{H S}=\left[\begin{array}{cc}
N_{m}(i \alpha) & D \\
0 & -N_{m}(i \alpha)^{H}
\end{array}\right]
$$

where $m=\left(r_{1}+r_{2}\right) / 2, d=\left(r_{1}-r_{2}\right) / 2$ and $D=\tau e_{d} e_{m}^{H}+\bar{\tau} e_{m} e_{d}^{H}, \tau= \pm i / 2$ if $r_{1}$ is odd and $\tau= \pm 1 / 2$ if $r_{2}$ is even. All Lagrangian invariant subspaces of $\mathcal{H}$ can be parametrized by

$$
\text { range } \mathcal{S}\left[\begin{array}{cc}
I_{t} & 0 \\
0 & W \\
0 & 0 \\
0 & -I_{s}
\end{array}\right]
$$

and all symplectic matrices that transform $\mathcal{H}$ to Hamiltonian block triangular form can be parametrized as

$$
\mathcal{S}\left[\begin{array}{cccc}
I_{p} & 0 & 0 & 0 \\
0 & W & 0 & I_{q} \\
0 & 0 & I_{p} & 0 \\
0 & -I_{q} & 0 & 0
\end{array}\right] \mathcal{Y}
$$

where $\mathcal{Y}$ is symplectic block triangular, $d \leq t \leq m, t+s=m, W=W^{H}$ satisfying

$$
N_{s} W+W N_{s}^{H}=0
$$

which has infinitely many solutions for every $s>0$.

Proof. Note that $r_{1}+r_{2}$ is the size of the Hamiltonian matrix $\mathcal{H}$, which must be even. So $r_{1}$ and $r_{2}$ must be both even or odd. The canonical form and the form of the parameterization follows directly form Theorem 3.4 by setting there $p=1$. So we only need to prove that $d \leq t \leq m$ and that (3.12) holds. For $p=1,(3.8)$ reduces to

$$
N_{s} W+W N_{s}^{H}=K,
$$

where $K$ is the trailing $s \times s$ block of $D$. Then $K=0$ if $t \geq d\left(s \leq r_{2}\right)$ and $K=\tau e_{d-t} e_{s}^{H}+\bar{\tau} e_{s} e_{d-t}^{H}$ if $t<d\left(s>r_{2}\right)$. If $t \geq d$, then the singular Lyapunov equation has infinitely many Hermitian solutions $W$, see [11, 14]. If $t<d$ and $r_{1}$, $r_{2}$ are both even, then it follows that $\tau \neq 0$ is real. By comparing the elements, it follows that the Lyapunov equation has no solution. The same conclusion follows for the case that $r_{1}, r_{2}$ are both odd. Consequently $W$ exists if and only if $d \leq t \leq m$.

In this simple case the parameters are completely given. But more importantly this result also gives a sufficient condition that a Hamiltonian matrix has infinitely many Lagrangian invariant subspaces.

COROLlary 3.6. If a Hamiltonian matrix $\mathcal{H}$ has exactly one eigenvalue i $\alpha$ and has at least two even or two odd size Jordan blocks with opposite structure inertia indices, then $\mathcal{H}$ has infinitely many Lagrangian invariant subspaces. 
Proof. We may assume w.l.o.g. that the two (even or odd) Jordan blocks are arranged in trailing position of $\mathcal{R}$ in the canonical form (3.5). Choosing $t_{j}=r_{j}$ for all $j=1, \ldots, p-1$ implies that all $V_{i, j}$ are void, $W=W_{p, p}$ and

$$
U=\left[\begin{array}{ccc}
I & 0 & 0 \\
0 & I_{t_{p}} & 0 \\
0 & 0 & W_{p p} \\
0 & 0 & 0 \\
0 & 0 & -I_{s_{p}}
\end{array}\right]
$$

By Lemma 3.5 there are infinitely many Lagrangian invariant subspaces (that are parametrized by $W_{p, p}$ ) for the small Hamiltonian matrix

$$
H_{p}:=\left[\begin{array}{cc}
N_{m_{p}}(i \alpha) & D_{p} \\
0 & -N_{m_{p}}(i \alpha)^{H}
\end{array}\right]
$$

and hence, there are also infinitely many Lagrangian invariant subspaces for $\mathcal{H}$.

This corollary shows that to obtain a unique Lagrangian invariant subspace all structure inertia indices of $\mathcal{H}$ have to have the same sign. Moreover, by Theorem 2.2 this also implies that $\mathcal{H}$ has only even size Jordan blocks. In the next section we will prove that this is also sufficient.

In order to complete the analysis we need to study Hamiltonian matrices $\mathcal{H}$ that have only two eigenvalues $\lambda,-\bar{\lambda}$ that are not purely imaginary. If $\mathcal{H} \in \mathbf{C}^{2 n, 2 n}$ then the algebraic multiplicities of $\lambda,-\bar{\lambda}$ are both $n$ and hence $\Omega(\mathcal{H})$ consists of $n+1$ selections $\omega(m), m=0, \ldots, n$, where $\omega(m)$ contains $m$ copies of $\lambda$ and $n-m$ copies of $-\bar{\lambda}$.

It follows from Theorem 2.2 that in this case there exists a symplectic matrix $\mathcal{S}$ such that

$$
\mathcal{R}:=\mathcal{S}^{-1} \mathcal{H S}=\left[\begin{array}{cc}
N(\lambda) & 0 \\
0 & -N(\lambda)^{H}
\end{array}\right]
$$

where $N(\lambda)=\lambda I+N, N=\operatorname{diag}\left(N_{r_{1}}, \ldots, N_{r_{p}}\right)$.

For every $\omega(m), 0 \leq m \leq n$, the parameterization of all possible Lagrangian invariant subspaces can be derived in a similar way as in the case of purely imaginary eigenvalues.

THEOREM 3.7. Let $\mathcal{H} \in \mathbf{C}^{2 n \times 2 n}$ be a Hamiltonian matrix that has only eigenvalues $\lambda,-\bar{\lambda}$ which are not purely imaginary. Let $\mathcal{S}$ be a symplectic matrix that transforms $\mathcal{H}$ to the form (3.13). For every selection $\omega(m) \in \Omega(\mathcal{H})$ all the corresponding 
invariant subspaces can be parametrized by range $\mathcal{S} U$, where $U$ has the form

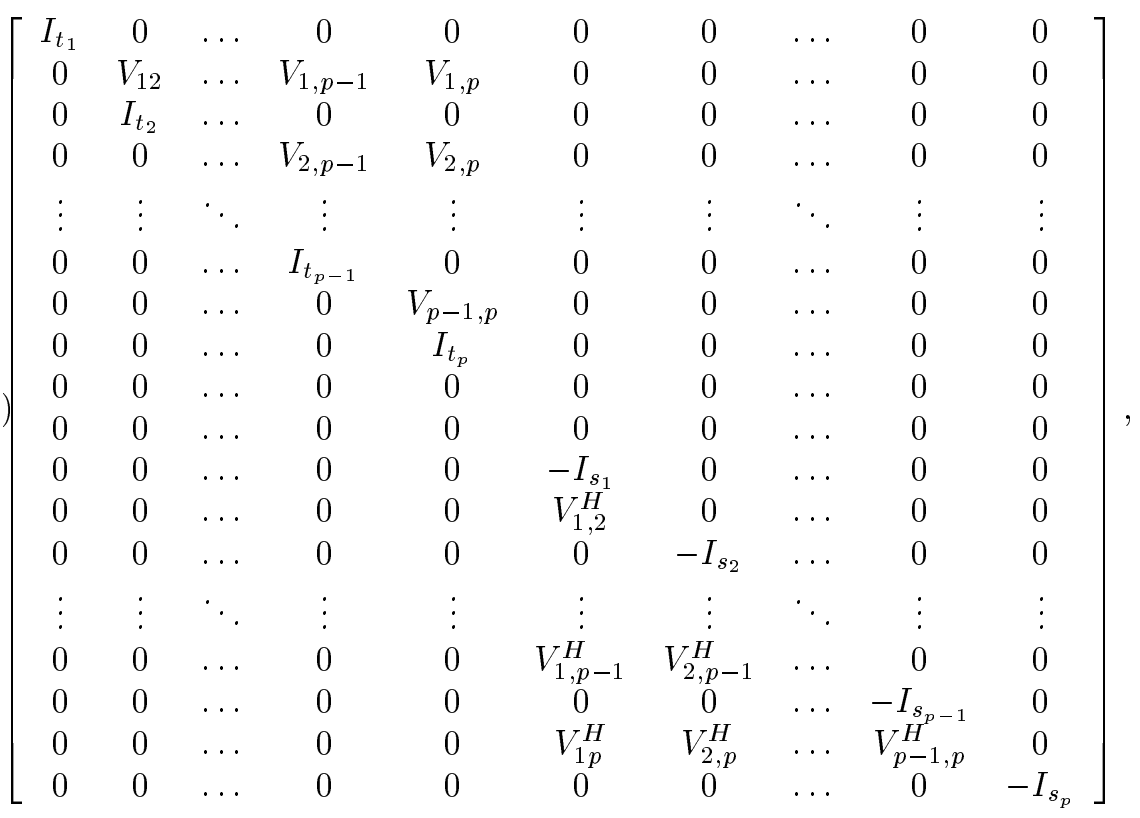

with $0 \leq s_{j}, t_{j} \leq r_{j}, s_{j}+t_{j}=r_{j}$ and $\sum_{j=1}^{p} t_{j}=m$. If we set

$$
\begin{aligned}
M_{t} & =\operatorname{diag}\left(N_{t_{1}}, \ldots, N_{t_{p}}\right), \\
M_{s} & =\operatorname{diag}\left(N_{s_{1}}, \ldots, N_{s_{p}}\right), \\
E & =\operatorname{diag}\left(e_{t_{1}} e_{1}^{H}, \ldots, e_{t_{p}} e_{1}^{H}\right),
\end{aligned}
$$

then the matrix

$$
V:=\left[\begin{array}{cccc}
0 & V_{12} & \ldots & V_{1 p} \\
& \ddots & \ddots & \vdots \\
& & \ddots & V_{p-1, p} \\
& & & 0
\end{array}\right]
$$

must satisfy the Riccati equation

$$
0=M_{s} V-V M_{t}-V E V
$$

Every Lagrangian invariant subspace associated with $\omega(m)$ is uniquely determined by a set of parameters $\left\{t_{1}, \ldots, t_{p}\right\}$ with $0 \leq t_{j} \leq r_{j}$ and $\sum_{j=1}^{p} t_{j}=m$, and a set of matrices $V_{i, j}, i=1, \ldots, p-1, j=i+1, \ldots, p$ satisfying (3.15).

Moreover, all symplectic matrices that transform $\mathcal{H}$ to Hamiltonian block triangular form can be parametrized by $\mathcal{S U Y}$, where $\mathcal{Y}$ is symplectic block triangular, 
$\mathcal{U}=[U, \tilde{U}]$ with $U$ as in (3.10) and

$$
\tilde{U}=\left[\begin{array}{cccccccccc}
0 & 0 & \ldots & 0 & 0 & 0 & 0 & \ldots & 0 & 0 \\
0 & 0 & \ldots & 0 & 0 & I_{t_{1}} & 0 & \ldots & 0 & 0 \\
0 & 0 & \ldots & 0 & 0 & 0 & 0 & \ldots & 0 & 0 \\
0 & 0 & \ldots & 0 & 0 & 0 & I_{t_{2}} & \ldots & 0 & 0 \\
\vdots & \vdots & \ddots & \vdots & \vdots & \vdots & \vdots & \ddots & \vdots & \vdots \\
0 & 0 & \ldots & 0 & 0 & 0 & 0 & \ldots & 0 & 0 \\
0 & 0 & \ldots & 0 & 0 & 0 & 0 & \ldots & I_{t_{p-1}} & 0 \\
0 & 0 & \ldots & 0 & 0 & 0 & 0 & \ldots & 0 & 0 \\
0 & 0 & \ldots & 0 & 0 & 0 & 0 & \ldots & 0 & I_{t_{p}} \\
I_{s_{1}} & 0 & \ldots & 0 & 0 & 0 & 0 & \ldots & 0 & 0 \\
0 & 0 & \ldots & 0 & 0 & 0 & 0 & \ldots & 0 & 0 \\
0 & I_{s_{2}} & \ldots & 0 & 0 & 0 & 0 & \ldots & 0 & 0 \\
0 & 0 & \ldots & 0 & 0 & 0 & 0 & \ldots & 0 & 0 \\
\vdots & \vdots & \ddots & \vdots & \vdots & \vdots & \vdots & \ddots & \vdots & \vdots \\
0 & 0 & \ldots & I_{s_{p-1}} & 0 & 0 & 0 & \ldots & 0 & 0 \\
0 & 0 & \ldots & 0 & 0 & 0 & 0 & \ldots & 0 & 0 \\
0 & 0 & \ldots & 0 & I_{s_{p}} & 0 & 0 & \ldots & 0 & 0 \\
0 & 0 & \ldots & 0 & 0 & 0 & 0 & \ldots & 0 & 0
\end{array}\right] .
$$

Proof. It is sufficient to consider the Lagrangian invariant subspaces of $\mathcal{R}$ in (3.13). Let the columns of $X$ span a Lagrangian invariant subspace of $\mathcal{R}$ associated with $\omega(m)$. Then $\mathcal{R} X=X A$ and $\Lambda(A)=\omega(m)$. Since $\lambda \neq-\bar{\lambda}$, there exists a matrix $Y$ such that $Y^{-1} A Y=\left[\begin{array}{cc}A_{1} & 0 \\ 0 & A_{2}\end{array}\right]$, where $A_{1}$ is $m \times m$ and has only the eigenvalue $\lambda$ and $A_{2}$ is $(n-m) \times(n-m)$ and has only the eigenvalue $-\bar{\lambda}$. If we partition $X Y=\left[\begin{array}{ll}X_{11} & X_{12} \\ X_{21} & X_{22}\end{array}\right]$ conformally with $Y^{-1} A Y$, then from the block diagonal form of $\mathcal{R}$ we obtain $X_{12}=0, X_{21}=0$ and $N(\lambda) X_{11}=X_{11} A_{1},-N(\lambda)^{H} X_{22}=X_{22} A_{2}$, since $X_{11}, X_{22}$ must have full column rank. We apply Lemma 3.2 and then the result follows as in the case of purely imaginary eigenvalues.

The parametrization in this case is essentially the same as in case of purely imaginary eigenvalues except that in this case $W$ is void and that $\sum_{j=1}^{p} t_{j}$ is fixed for a given $\omega(m)$. In both cases the blocks $V_{i, j}$ still satisfy a sequence of Sylvester equations (3.11).

Again we have a corollary.

Corollary 3.8. Let $\mathcal{H} \in \mathbf{C}^{2 n \times 2 n}$ be a Hamiltonian matrix that has only the eigenvalues $\lambda,-\bar{\lambda}$ which are not purely imaginary. If $\mathcal{H}$ has exactly two Jordan blocks with respect to $\lambda$, then for every fixed $\omega(m) \in \Omega(\mathcal{H})$ the corresponding Lagrangian invariant subspaces can be paramterized as

$$
\mathcal{S}\left[\begin{array}{cccc}
I_{t_{1}} & 0 & 0 & 0 \\
0 & V & 0 & 0 \\
0 & I_{t_{2}} & 0 & 0 \\
0 & 0 & 0 & 0 \\
0 & 0 & 0 & 0 \\
0 & 0 & -I_{s_{1}} & 0 \\
0 & 0 & V^{H} & 0 \\
0 & 0 & 0 & -I_{s_{2}}
\end{array}\right]
$$


where $t_{1}+t_{2}=m, t_{j}+s_{j}=r_{j}$ and $0 \leq t_{j}, s_{j} \leq r_{j}$ for $j=1,2$.

Furthermore, $V=[0, T]$ if $s_{1}<t_{2}$ and $V=\left[\begin{array}{l}T \\ 0\end{array}\right]$ if $s_{1} \geq t_{2}$, where $T$ is an arbitrary square upper triangular Toeplitz matrix. So for every $\omega(m)$ with $0<m<n$ there are infinitely many Lagrangian invariant subspaces.

Proof. Applying Theorem 3.7 for $p=2$ we obtain the parametrization and the restrictions for $t_{1}, t_{2}$. The expression for $V$ follows from the fact that $V$ satisfies the Sylvester equation $N_{s_{1}} V-V N_{t_{2}}=0$.

In this special case we have the following uniqueness result.

Corollary 3.9. Let $\mathcal{H} \in \mathbf{C}^{2 n \times 2 n}$ be a Hamiltonian matrix that has only the eigenvalues $\lambda,-\bar{\lambda}$ which are not purely imaginary. Then we have the following:

i) For $\omega(0)$ or $\omega(n)$ the corresponding Lagrangian subspace is unique.

ii) If $\mathcal{H}$ has only a single Jordan block with respect to $\lambda$ then for every fixed $\omega(m) \in \Omega(\mathcal{H})$ with $0 \leq m \leq n$ the corresponding Lagrangian invariant subspace is unique. In this case there exists a symplectic matrix $\hat{\mathcal{S}}$ such that

$$
\hat{\mathcal{S}}^{-1} \mathcal{H} \hat{\mathcal{S}}=\left[\begin{array}{cc}
R & D \\
0 & -R^{H}
\end{array}\right]
$$

with $R=\operatorname{diag}\left(N_{m}(\lambda),-N_{n-m}(\lambda)^{H}\right), D=e_{m} e_{m+1}^{H}+e_{m+1} e_{m}^{H}$.

iii) If $\mathcal{H}$ has at least two Jordan blocks with respect to $\lambda$, then for every fixed $\omega(m) \in \Omega(\mathcal{H})$ with $0<m<n$ there are infinitely many corresponding Lagrangian invariant subspaces.

Proof. i) For $\omega(0)$ all $t_{j}$ must be zero so $U=\left[\begin{array}{c}0 \\ -I_{n}\end{array}\right]$ is unique. Analogously, for $\omega(n)$ the unique Lagrangian invariant subspace is $U=\left[\begin{array}{c}I_{n} \\ 0\end{array}\right]$.

ii) By assumption $p=1$, so for a fixed $\omega(m), U$ is unique as $\left[\begin{array}{c}I_{m} \\ 0 \\ 0 \\ -I_{n-m}\end{array}\right]$. Then

(3.16) follows from (3.13) and the special form $\mathcal{U}$ for $p=1$.

iii) In this case we can choose the integers $t_{j}$ such that $t_{1}<r_{1}$ and $t_{p}>0$. We set $V_{i, j}=0$ except for $V_{1, p}$ which is chosen to satisfy $N_{s_{1}} V_{1, p}-V_{1, p} N_{t_{p}}=0$. Since $s_{1}, t_{p}>0$, there are infinitely many solutions $V_{1, p}$ and hence, infinitely many $U$.

In the next section we will use the parametrizations to characterize existence and uniqueness of Lagrangian invariant subspaces.

4. Existence and uniqueness of Lagrangian invariant subspaces. In this section we summarize all results given in the previous sections and give a complete characterization of the existence and the uniqueness of Lagrangian invariant subspaces for a Hamiltonian matrix. This complete result includes previous results based on structure inertia indices of $[23,25]$.

TheOREM 4.1. (Existence) Let $\mathcal{H} \in \mathbf{C}^{2 n \times 2 n}$ be a Hamiltonian matrix, let $i \alpha_{1}, \ldots, i \alpha_{\nu}$ be its pairwise distinct purely imaginary eigenvalues and let $\lambda_{1},-\bar{\lambda}_{1}, \ldots$, $\lambda_{\mu},-\bar{\lambda}_{\mu}$ be its pairwise distinct nonimaginary eigenvalues. The following are equivalent:

i) $\mathcal{H}$ has a Lagrangian invariant subspace for one $\omega \in \Omega(\mathcal{H})$.

ii) $\mathcal{H}$ has a Lagrangian invariant subspace for all $\omega \in \Omega(\mathcal{H})$.

iii) There exists a symplectic matrix $\mathcal{S}$, such that $\mathcal{S}^{-1} \mathcal{H} \mathcal{S}$ is Hamiltonian block triangular. 
iv) There exists a unitary symplectic matrix $\mathcal{U}$, such that $\mathcal{U}^{H} \mathcal{H} \mathcal{U}$ is Hamiltonian block triangular.

v) For all $k=1, \ldots, \nu$, if $U_{k}$ span the invariant subspace associated with $i \alpha_{k}$, then $U_{k}^{H} J U_{k}$ is congruent to $J_{m_{k}}$.

vi) $\operatorname{Ind}_{S}^{d}\left(i \alpha_{k}\right)$ is void for all $k=1, \ldots, \nu$.

Proof. This result in different notation is known, see [19, 23, 24, 25].

THEOREM 4.2. (Uniqueness for $\tilde{\Omega}(\mathcal{H})$ ). Let $\mathcal{H} \in \mathbf{C}^{2 n \times 2 n}$ be a Hamiltonian matrix. Let $i \alpha_{1}, \ldots, i \alpha_{\nu}$ be its pairwise distinct purely imaginary eigenvalues and let $\lambda_{1},-\bar{\lambda}_{1}, \ldots, \lambda_{\mu},-\bar{\lambda}_{\mu}$ be its pairwise distinct nonimaginary eigenvalues. Suppose that any of the equivalent conditions of Theorem 4.1 for the existence of Lagrangian invariant subspaces holds. Then the following are equivalent:

i) For every $\omega \in \tilde{\Omega}(\mathcal{H})$ there exists a unique associated Lagrangian invariant subspace.

ii) If $\omega \in \tilde{\Omega}(\mathcal{H})$ and if $\mathcal{S}_{1}$ and $\mathcal{S}_{2}$ are symplectic matrices such that $\mathcal{S}_{1}^{-1} \mathcal{H S}_{1}=$ $\left[\begin{array}{cc}R_{1} & D_{1} \\ 0 & -R_{1}^{H}\end{array}\right], \mathcal{S}_{2}^{-1} \mathcal{H} \mathcal{S}_{2}=\left[\begin{array}{cc}R_{2} & D_{2} \\ 0 & -R_{2}^{H}\end{array}\right]$ and $\Lambda\left(R_{1}\right)=\Lambda\left(R_{2}\right)=\omega$, then $\mathcal{S}_{1}^{-1} \mathcal{S}_{2}$ is symplectic block triangular.

iii) There exists an $\omega \in \tilde{\Omega}(\mathcal{H})$ such that $\mathcal{H}$ has a unique associated Lagrangian invariant subspace.

iv) There exists an $\omega \in \tilde{\Omega}(\mathcal{H})$ such that if $\mathcal{S}_{1}$ and $\mathcal{S}_{2}$ are symplectic matrices satisfying $\mathcal{S}_{1}^{-1} \mathcal{H} \mathcal{S}_{1}=\left[\begin{array}{cc}R_{1} & D_{1} \\ 0 & -R_{1}^{H}\end{array}\right], \mathcal{S}_{2}^{-1} \mathcal{H S}_{2}=\left[\begin{array}{cc}R_{2} & D_{2} \\ 0 & -R_{2}^{H}\end{array}\right]$ and $\Lambda\left(R_{1}\right)=\Lambda\left(R_{2}\right)=\omega$, then $\mathcal{S}_{1}^{-1} \mathcal{S}_{2}$ is symplectic block triangular.

v) Let $\left[\begin{array}{cc}A & B \\ 0 & -A^{H}\end{array}\right]$ be an arbitrary Hamiltonian block triangular form of $\mathcal{H}$. If for a purely imaginary eigenvalue $i \alpha_{k}$ the columns of $\Phi_{k}$ form a basis of the left eigenvector subspace of $A$, i.e., $\Phi_{k}^{H} A=i \alpha_{k} \Phi_{k}^{H}$, then $\Phi_{k}^{H} B \Phi_{k}$ is positive definite or negative definite.

vi) For every purely imaginary eigenvalue i $\alpha_{k}$ there are only even sized Jordan blocks which, furthermore, have all structure inertia indices of the same sign.

If the uniqueness conditions do not hold, then for every $\omega \in \tilde{\Omega}(\mathcal{H})$ there are infinitely many Lagrangian invariant subspaces. They can be parametrized by applying Theorem 3.4 for every $i \alpha_{k}$.

Proof. The proof of the equivalence of i) and vi) has been given (in different notation) in Theorem 1.3 of [25]. For completeness we give the whole proof in our terminology. By the argument in Section 3 it suffices to consider a Hamiltonian matrix $\mathcal{H}$ that has either a single purely imaginary eigenvalue $i \alpha$ or an eigenvalue pair $\lambda$ and $-\bar{\lambda}$. In the first case we again take $i \alpha=0$.

Since by Corollary 3.9 for nonimaginary eigenvalues the corresponding invariant subspaces are unique, we only need to consider the case of a purely imaginary eigenvalue.

The proofs of i) $\Leftrightarrow$ ii) and iii) $\Leftrightarrow$ iv) are obvious. Corollary 3.6 implies that ii) $\Rightarrow$ vi). If vi) holds, then by Theorem 2.2 there exists a symplectic matrix $\mathcal{S}$ such that

$$
\mathcal{R}:=\mathcal{S}^{-1} \mathcal{H S}=\left[\begin{array}{cc}
R & D \\
0 & -R^{H}
\end{array}\right]
$$

where $R=\operatorname{diag}\left(N_{l_{1}}, \ldots, N_{l_{q}}\right)$ and $D=\beta \operatorname{diag}\left(e_{l_{1}} e_{l_{1}}^{H}, \ldots, e_{l_{q}} e_{l_{q}}^{H}\right)$. (Recall that $\left.i \alpha=0.\right)$ We only need to prove that for every symplectic $\mathcal{Z}$ satisfying $\mathcal{Z}^{-1} \mathcal{R} \mathcal{Z}=\left[\begin{array}{cc}\tilde{R} & \tilde{D} \\ 0 & -\tilde{R}^{H}\end{array}\right]$, 
$\mathcal{Z}$ is block triangular. Partitioning $\mathcal{Z}=\left[\begin{array}{ll}Z_{11} & Z_{12} \\ Z_{21} & Z_{22}\end{array}\right]$, it follows that

$$
R Z_{11}+D Z_{21}=Z_{11} \tilde{R}
$$

and

$$
-R^{H} Z_{21}=Z_{21} \tilde{R}
$$

Suppose that $Z_{21} \neq 0$, then it follows by (4.3) that range $Z_{21}$ is an invariant subspace of $-R^{H}$. Hence, there exists a vector $x$ such that $Z_{21} x \neq 0$ and

$$
R^{H} Z_{21} x=0
$$

i.e., $Z_{21} x$ is a left eigenvector of $R$. Multiplying $\left(Z_{21} x\right)^{H}$ and $x$ on both sides of (4.2) and using (4.4) we get

$$
\left(Z_{21} x\right)^{H} D\left(Z_{21} x\right)=-x^{H} Z_{21}^{H} Z_{11} \tilde{R} x .
$$

Since $\mathcal{Z}$ is symplectic, we have $Z_{21}^{H} Z_{11}=Z_{11}^{H} Z_{21}$. Combining (4.3) and (4.4) we get

$$
x^{H} Z_{21}^{H} Z_{11} \tilde{R} x=x^{H} Z_{11}^{H} Z_{21} \tilde{A} x=-x^{H} Z_{11}^{H} R^{H} Z_{21} x=0
$$

and, therefore,

$$
\left(Z_{21} x\right)^{H} D\left(Z_{21} x\right)=0 .
$$

On the other hand, since $Z_{21} x$ is a left eigenvector of $R$, by the structure of $R$ there must exist a nonzero vector $y$ such that $Z_{21} x=E y$, where

$$
E:=\left[e_{p_{1}}, \ldots, e_{p_{q}}\right] \text {, }
$$

with $p_{k}=\sum_{j=1}^{k} l_{j}$ for $k=1, \ldots, q$. But $E^{H} D E=\beta I_{q}$ and hence

$$
0=\left(Z_{21} x\right)^{H} D\left(Z_{21} x\right)=y^{H} E^{H} D E y=\beta y^{H} y \neq 0,
$$

which is a contradiction. $\Leftrightarrow$ i).

i) $\Rightarrow$ iii) is obvious and iii) $\Rightarrow$ i) follows from iii) $\Rightarrow$ vi) by Corollary 3.6 and vi)

To prove vi $\Rightarrow$ v) let $\hat{\mathcal{R}}=\left[\begin{array}{cc}A & B \\ 0 & -A^{H}\end{array}\right]$ be an arbitrary Hamiltonian triangular form of $\mathcal{H}$ and let $\mathcal{R}$ be as in (4.1). Since vi) holds and vi) $\Leftrightarrow$ ii), there exists a symplectic block triangular matrix $\mathcal{S}=\left[\begin{array}{cc}S_{1} & S_{2} \\ 0 & S_{1}^{-H}\end{array}\right]$ (see [5]) such that $\hat{\mathcal{R}}=\mathcal{S}^{-1} \mathcal{R} \mathcal{S}$. Hence $S_{1}^{-1} R S_{1}=A$ and $B=S_{1}^{-1} R S_{2}+S_{1}^{-1} D S_{1}^{-H}+S_{2}^{H} R^{H} S_{1}^{-H}$. Since $A$ is similar to $R$, a left eigenvector subspace of $A$ can be chosen as $\Phi=S_{1}^{H} E$, where $E$ is as in (4.5). Then a simple calculation yields $\Phi^{H} B \Phi=\beta I_{q}$.

For v) $\Rightarrow$ vi) suppose that $\hat{\mathcal{R}}=\left[\begin{array}{cc}A & B \\ 0 & -A^{H}\end{array}\right]$ satisfies v). Using the same argument as for vi) $\Rightarrow$ ii) and replacing $\mathcal{R}$ by $\hat{\mathcal{R}}$ we obtain that v) $\Rightarrow$ ii). Since ii) $\Leftrightarrow$ vi), it follows that v) also implies vi).

Theorem 4.3. (Uniqueness for $\Omega(\mathcal{H})$ ). Let $\mathcal{H} \in \mathbf{C}^{2 n \times 2 n}$ be a Hamiltonian matrix. Let $i \alpha_{1}, \ldots, i \alpha_{\nu}$ be its pairwise distinct purely imaginary eigenvalues and let $\lambda_{1},-\bar{\lambda}_{1}, \ldots, \lambda_{\mu},-\bar{\lambda}_{\mu}$ be its pairwise distinct nonimaginary eigenvalues. Suppose that any of the equivalent conditions of Theorem 4.1 for the existence of Lagrangian invariant subspaces holds. Then the following are equivalent: 
i) For every $\omega \in \Omega(\mathcal{H})$ there exists a unique associated Lagrangian invariant subspace.

ii) Let $\omega \in \Omega(\mathcal{H})$. If $\mathcal{S}_{1}$ and $\mathcal{S}_{2}$ are symplectic matrices such that $\mathcal{S}_{1}^{-1} \mathcal{H S}_{1}=$ $\left[\begin{array}{cc}R_{1} & D_{1} \\ 0 & -R_{1}^{H}\end{array}\right], \mathcal{S}_{2}^{-1} \mathcal{H S}_{2}=\left[\begin{array}{cc}R_{2} & D_{2} \\ 0 & -R_{2}^{H}\end{array}\right]$ and $\Lambda\left(R_{1}\right)=\Lambda\left(R_{2}\right)=\omega$, then $\mathcal{S}_{1}^{-1} \mathcal{S}_{2}$ is symplectic block triangular.

iii) There exists an $\omega \in \Omega(\mathcal{H})$ but $\omega \notin \tilde{\Omega}(\mathcal{H})$ such that $\mathcal{H}$ has a unique associated Lagrangian invariant subspace.

iv) There exists an $\omega \in \Omega(\mathcal{H})$ but $\omega \notin \tilde{\Omega}(\mathcal{H})$ such that if $\mathcal{S}_{1}$ and $\mathcal{S}_{2}$ are symplectic matrices satisfying $\mathcal{S}_{1}^{-1} \mathcal{H} \mathcal{S}_{1}=\left[\begin{array}{cc}R_{1} & D_{1} \\ 0 & -R_{1}^{H}\end{array}\right], \mathcal{S}_{2}^{-1} \mathcal{H S}_{2}=\left[\begin{array}{cc}R_{2} & D_{2} \\ 0 & -R_{2}^{H}\end{array}\right]$ and $\Lambda\left(R_{1}\right)=\Lambda\left(R_{2}\right)=\omega$, then $\mathcal{S}_{1}^{-1} \mathcal{S}_{2}$ is symplectic block triangular.

v) Let $\left[\begin{array}{cc}A & B \\ 0 & -A^{H}\end{array}\right]$ be an arbitrary Hamiltonian block triangular form of $\mathcal{H}$. Then $A$ either has one of $\lambda_{k},-\bar{\lambda}_{k}$ as its eigenvalue and has a unique corresponding left eigenvector, or $A$ has both $\lambda_{k},-\bar{\lambda}_{k}$ as eigenvalues and has unique corresponding left eigenvectors $x_{k}$ and $y_{k}$ such that $x_{k}^{H} B y_{k} \neq 0$. Furthermore, for every $i \alpha_{k}$ if the columns of $\Phi_{k}$ form a basis of the left eigenvector subspace of A, i.e., $\Phi_{k}^{H} A=i \alpha_{k} \Phi_{k}^{H}$, then $\Phi_{k}^{H} B \Phi_{k}$ is positive definite or negative definite.

vi) For every nonimaginary eigenvalue, $\mathcal{H}$ has only one corresponding Jordan block and for every purely imaginary eigenvalue $i \alpha_{k}, \mathcal{H}$ has only even sized Jordan blocks with all structure inertia indices of the same sign.

If the uniqueness conditions do not hold, then for every $\omega \in \Omega(\mathcal{H})$ there are infinitely many Lagrangian invariant subspaces. They can be parametrized by applying Theorem 3.4 for every $i \alpha_{k}$ and Theorem 3.7 for every pair $\lambda_{k},-\bar{\lambda}_{k}$.

Proof. The proof of the equivalence of i) and vi) has again been given (in different notation) in Theorem 1.3 of [25]. For completeness we again give the whole proof in our terminology.

By the argument in Section 3 it suffices to consider that the Hamiltonian matrix $\mathcal{H}$ has only either a single purely imaginary eigenvalue $i \alpha$ or an eigenvalue pair $\lambda$ and $-\bar{\lambda}$, and in the first case we will assume $i \alpha=0$. For the purely imaginary eigenvalue the proof is as that of Theorem 4.2. Hence, consider $\mathcal{H}$ with an eigenvalue pair $\lambda,-\lambda$. The parts i) $\Leftrightarrow$ ii) and iii) $\Leftrightarrow$ iv) are obvious. i) $\Leftrightarrow$ vi) follows from Corollary 3.9. i) $\Leftrightarrow$ iii) follows, since iii) $\Rightarrow$ vi) and vi) $\Leftrightarrow$ i), since $\omega \notin \tilde{\Omega}(\mathcal{H})$ implies that both $\lambda$ and $-\bar{\lambda}$ have been chosen in $\omega$. It remains to prove $\mathrm{v}) \Leftrightarrow \mathrm{vi}$ ). We may assume that both $\lambda,-\bar{\lambda}$ are in $\Lambda(A)$, since otherwise $\omega \in \tilde{\Omega}(\mathcal{H})$.

For vi) $\Rightarrow \mathrm{v}$ ) let $\hat{\mathcal{R}}=\left[\begin{array}{cc}A & B \\ 0 & -A^{H}\end{array}\right]$ be an arbitrary Hamiltonian triangular form of $\mathcal{H}$. Since vi) holds, by (3.16) in Corollary 3.9 the Hamiltonian canonical form is $\mathcal{R}=\left[\begin{array}{cc}R & D \\ 0 & -R^{H}\end{array}\right]$, where $R=\operatorname{diag}\left(N_{t}(\lambda),-N_{s}(\lambda)^{H}\right), D=e_{t} e_{t+1}^{H}+e_{t+1} e_{t}^{H}$ and $t$ is the multiplicity of $\lambda$ in $\Lambda(A)$. By ii) there exists a symplectic matrix $\mathcal{S}=\left[\begin{array}{cc}S_{1} & S_{2} \\ 0 & S_{1}^{-H}\end{array}\right]$ such that $\hat{\mathcal{R}}=\mathcal{S}^{-1} \mathcal{R S}$. Hence $S_{1}^{-1} R S_{1}=A$ and $B=S_{1}^{-1} R S_{2}+S_{1}^{-1} D S_{1}^{-H}+S_{2}^{H} R^{H} S_{1}^{-H}$. If only one of $\lambda,-\bar{\lambda}$ is in $\Lambda(A)$, then, since $A$ is similar to $R$, it is also in $\Lambda(R)$. Hence, either $t=0$ or $s=0$ and $R$ (and thus also $A$ ) has only one corresponding left eigenvector. If both $\lambda,-\bar{\lambda}$ are in $\Lambda(A)$ then $s, t>0$. In this case $R$ has only left eigenvectors $e_{t}$ and $e_{t+1}$ with respect to $\lambda$ and $-\bar{\lambda}$, respectively. Therefore, $A$ also has only left eigenvectors $S_{1}^{H} e_{t}$ and $S_{1}^{H} e_{t+1}$, for $\lambda$ and $-\bar{\lambda}$, respectively. Then it is easy to see that $e_{t}^{H} S_{1} B S_{1} e_{t+1}=e_{t}^{H} D e_{t+1}=1$.

For v) $\Rightarrow$ vi), if $A$ has only one of $\lambda,-\bar{\lambda}$ as its eigenvalue and has a unique left eigenvector, then $A$ also has only one right eigenvector. Since $\Lambda(A) \cap \Lambda\left(-A^{H}\right)=\emptyset$ 
in this case $\hat{\mathcal{R}}$ has also a unique corresponding right eigenvector. Therefore, there is only one corresponding Jordan block. By the canonical form (3.13) for the another eigenvalue there is also only one Jordan block. If $A$ has both $\lambda,-\bar{\lambda}$, then we first show that for left eigenvectors $x, y$ of $A$ such that $x^{H} B y \neq 0$ condition ii) holds. Then we show that $x, y$ must be unique. As in the proof of Theorem 4.2 we only need to prove that for every symplectic matrix $\mathcal{Z}$ satisfying $\mathcal{Z}^{-1} \hat{\mathcal{R}} \mathcal{Z}=\left[\begin{array}{cc}\tilde{R} & \tilde{D} \\ 0 & -\tilde{R}^{H}\end{array}\right], \Lambda(\tilde{R})=\Lambda(A)$ it follows that $\mathcal{Z}$ is block triangular. Partitioning $\mathcal{Z}=\left[\begin{array}{ll}Z_{11} & Z_{12} \\ Z_{21} & Z_{22}\end{array}\right]$, it follows that

$$
A Z_{11}+B Z_{21}=Z_{11} \tilde{R}
$$

and

$$
-A^{H} Z_{21}=Z_{21} \tilde{R}
$$

Suppose that $Z_{21} \neq 0$, then range $Z_{21}$ is an invariant subspace of $-A^{H}$. Hence there exists $z_{1}$, with either $\tilde{R} z_{1}=-\bar{\lambda} z_{1}$ or $\tilde{R} z_{1}=\lambda z_{1}$ such that $Z_{21} z_{1} \neq 0$, which implies that $Z_{21} z_{1}$ is the left eigenvectors of $A$ corresponding to $\lambda$ or $-\bar{\lambda}$. W.l.o.g, assume that $z_{1}$ satisfies $\tilde{R} z_{1}=-\bar{\lambda} z_{1}$. Let $z_{2} \neq 0$ satisfy $z_{2}^{H} A=-\bar{\lambda} z_{2}^{H}$. Multiplying $z_{2}^{H}$ and $z_{1}$ on both sides of (4.6), a simple calculation yields $z_{1}^{H} B\left(Z_{21} z_{2}\right)=0$, which is a contradiction.

Suppose that $x, y$ are not unique. Then let $X$ form the left eigenvector subspace of $A$ with respect to $\lambda$. Since $X^{H} B y$ has more than one row there always exists a vector $z$ such that $z^{H} X^{H} B y=0$, which is a contradiction. So $x$ and $y$ must be unique.

Remark 2. For real Hamiltonian matrices it is reasonable to consider real Lagrangian invariant subspaces. For this problem we have to give a natural additional restriction on the eigenvalue selections. Note that in this case if $\lambda$ is a nonreal eigenvalue of $\mathcal{H}$, then $\bar{\lambda},-\bar{\lambda}$ and $-\lambda$ are also eigenvalues of $\mathcal{H}$. To obtain real invariant subspaces it is necessary to keep the associated eigenvalues in conjugate pairs. So if we choose a nonreal $\lambda$ we must choose $\bar{\lambda}$ with same multiplicity. But essentially we can use the same construction as for the complex case to solve this problem, see [19], since if $\mathcal{H}$ is real then for real eigenvalues the corresponding invariant subspaces can be chosen real. So for these eigenvalues we can still use Theorems 3.7 and 3.4 by chosing $V$ and $W$ real.

In this section we have given necessary conditions for the existence and uniqueness of Lagrangian invariant subspaces. In the following section we obtain as corollaries several results on the existence and uniqueness of Hermitian solutions of algebraic Riccati equations.

5. Hermitian solutions of Riccati equations. In this section we apply the existence and uniqueness results for Lagrangian invariant subspaces to analyze the existence and uniqueness of Hermitian solutions of algebraic Riccati equations

$$
A^{H} X+X A-X M X+G=0
$$

with $M=M^{H}$ and $G=G^{H}$. The related Hamiltonian matrix is $\mathcal{H}=\left[\begin{array}{cc}A & M \\ G & -A^{H}\end{array}\right]$. The following result is well-known, see, e.g., [15].

Proposition 5.1. The algebraic Riccati equation (5.1) has an Hermitian solution if and only if there exists a $2 n \times n$ matrix $L=\left[\begin{array}{l}L_{1} \\ L_{2}\end{array}\right]$, with $L_{1}, L_{2} \in \mathbf{C}^{n \times n}$ and $L_{1}$ 
invertible, such that the columns of $L$ span a Lagrangian invariant subspace of the related Hamiltonian matrix $\mathcal{H}$ associated to $\omega \in \Omega(\mathcal{H})$. In this case $X=-L_{2} L_{1}^{-1}$ is Hermitian and solves (5.1) and $\Lambda(A-M X)=\omega$.

It follows that we can study the existence and uniqueness of solutions of algebraic Riccati equations via the analysis of Lagrangian invariant subspaces of the associated Hamiltonian matrices.

Unlike the Lagrangian invariant subspace problem, which only depends on the Jordan structure, Hermitian solution of the Riccati equation depend further on the top block of the basis of the Lagrangian invariant subspace and the choice of the associated eigenvalues. In other words, for a given Hamiltonian block triangular form $\mathcal{R}$, all Hamiltonian matrices which are similar to $\mathcal{R}$ have Lagrangian invariant subspaces, while for Riccati equation solutions these Hamiltonian matrices may be partitioned into three groups which i) have Hermitian solutions for all selections $\Omega(\mathcal{R})$, ii) have Hermitian solutions for some $\omega \in \Omega(\mathcal{R})$, iii) have no Hermitian solution for any $\omega \in \Omega(\mathcal{R})$.

Example 1. Consider three Riccati equations with matrices
a) $A=\left[\begin{array}{ll}i & 0 \\ 0 & 1\end{array}\right], \quad M=\left[\begin{array}{cc}1 & -1-i \\ -1+i & 0\end{array}\right], \quad G=\left[\begin{array}{ll}0 & 0 \\ 0 & 0\end{array}\right]$,
b) $A=\left[\begin{array}{ll}i & 0 \\ 0 & 1\end{array}\right], \quad M=\left[\begin{array}{cc}1 & -1-i \\ -1+i & -2\end{array}\right], \quad G=\left[\begin{array}{ll}0 & 0 \\ 0 & 0\end{array}\right]$,
c) $A=\left[\begin{array}{cc}i & 0 \\ 0 & -1\end{array}\right], \quad M=\left[\begin{array}{ll}0 & 0 \\ 0 & 0\end{array}\right], \quad G=\left[\begin{array}{cc}-1 & -1+i \\ -1-i & 0\end{array}\right]$.

In all three cases the related Hamiltonian matrices have the same Hamiltonian Jordan canonical form

$$
\left[\begin{array}{cccc}
i & 0 & 1 & 0 \\
0 & 1 & 0 & 0 \\
0 & 0 & i & 0 \\
0 & 0 & 0 & -1
\end{array}\right]
$$

and $\Omega(\mathcal{H})=\left\{\omega_{1}, \omega_{2}\right\}$ with $\omega_{1}=\{i, 1\}, \omega_{2}=\{i,-1\}$. Certainly for both $\omega_{1}, \omega_{2}$ all Hamiltonian matrices have a unique Lagrangian invariant subspace. But the Hermitian solutions of Riccati equation are different. In case a) for $\omega_{1}$ the solution is $X=0$ and for $\omega_{2}$ there is no solution. In case b) for $\omega_{1}$ the solution is 0 and for $\omega_{2}$ the solution is $X=\left[\begin{array}{cc}0 & 0 \\ 0 & -1\end{array}\right]$. In case c) for both $\omega_{1}$ and $\omega_{2}$ there is no solution at all. It is also possible that the Riccati equation has no Hermitian solution while the related Hamiltonian matrix has infinitely many Lagrangian invariant subspaces.

EXAmple 2. For

$$
A=M=\left[\begin{array}{ll}
0 & 0 \\
0 & 0
\end{array}\right], \quad G=\left[\begin{array}{ll}
0 & 1 \\
1 & 0
\end{array}\right]
$$

the Riccati equation (5.1) has no solution. But for the associated Hamiltonian matrix the bases of the Lagrangian invariant subspace can be parametrized as

$$
\left[\begin{array}{cc}
-i \beta & 0 \\
-1 & 0 \\
0 & 1 \\
\alpha & i \beta
\end{array}\right], \quad\left[\begin{array}{ll}
0 & \gamma \\
0 & 0 \\
0 & 1 \\
1 & 0
\end{array}\right], \quad\left[\begin{array}{ll}
0 & 1 \\
0 & 0 \\
0 & 0 \\
1 & 0
\end{array}\right]
$$

where $\alpha, \beta, \gamma$ are real. 
By using the parameterizations in Section 3 we can give a necessary and sufficient condition for the existence of the Hermitian solutions of the Riccati equation (5.1). Note that for the solvability of the Riccati equation it is necessary that the Hamiltonian matrix $\mathcal{H}$ associated to (5.1) has a Hamiltonian block triangular form. So there exists a symplectic matrix $\mathcal{S}$ such that

$$
\mathcal{S}^{-1} \mathcal{H S}=\left[\begin{array}{cc}
R & D \\
0 & -R^{H}
\end{array}\right]
$$

with $R=\operatorname{diag}\left(R_{1}, \ldots, R_{\mu} ; R_{\mu+1}, \ldots, R_{\mu+\nu}\right), D=\operatorname{diag}\left(0, \ldots, 0 ; D_{\mu+1}, \ldots, D_{\mu+\nu}\right)$. A submatrix $H_{k}:=\left[\begin{array}{cc}R_{k} & 0 \\ 0 & -R_{k}^{H}\end{array}\right]$ has the Jordan form (3.13) with respect to the eigenvalues $\lambda_{k},-\bar{\lambda}_{k}$ for $k=1, \ldots, \mu$, and a submatrix $H_{k}:=\left[\begin{array}{cc}R_{k} & D_{k} \\ 0 & -R_{k}^{H}\end{array}\right]$ has the Jordan form (3.5) with respect to $i \alpha_{k-\mu}$ for $k=\mu+1, \ldots, \mu+\nu$.

THEOREM 5.2. Let $\mathcal{H}$ be the Hamiltonian matrix associated with the algebraic Riccati equation (5.1) and assume that $\mathcal{H}$ has a Hamiltonian block triangular form. Let $\mathcal{S}$ be a symplectic matrix satisfying (5.2) and let $\mathcal{P}$ be a permutation matrix such that

$$
\begin{aligned}
\mathcal{P}^{-1} \mathcal{S}^{-1} \mathcal{H} \mathcal{S P} & =\operatorname{diag}\left(H_{1}, \ldots, H_{\mu} ; H_{\mu+1}, \ldots, H_{\mu+\nu}\right), \\
\mathcal{P}^{H} J \mathcal{P} & =\operatorname{diag}\left(J_{n_{1}}, \ldots, J_{n_{\mu}} ; J_{m_{1}}, \ldots, J_{m_{\nu}}\right)
\end{aligned}
$$

where $H_{k}=\left[\begin{array}{cc}R_{k} & 0 \\ 0 & -R_{k}^{H}\end{array}\right]$. Then for an eigenvalue selection $\omega \in \Omega(\mathcal{H})$, the Riccati equation (5.1) has an Hermitian solution $X$ with $\Lambda(A-M X)=\omega$ if and only if there exist matrices $U_{1}, \ldots, U_{\mu}$ and $Q_{1}, \ldots, Q_{\nu}$ with the following properties. The matrices $U_{k}$ are $2 n_{k} \times n_{k}$ and have the block form (3.10) with blocks satisfying (3.15) and the matrices $Q_{k}$ are $2 m_{k} \times m_{k}$ and have the block form (3.6) with blocks satisfying (3.8) and (3.9), such that

$$
L_{1}:=\left[I_{n}, 0\right] \mathcal{S P} \operatorname{diag}\left(U_{1}, \ldots, U_{\mu} ; Q_{1}, \ldots, Q_{\nu}\right)
$$

is nonsingular.

Moreover, $X=-\left[0, I_{n}\right] \mathcal{S P} \operatorname{diag}\left(U_{1}, \ldots, U_{\mu} ; Q_{1}, \ldots, Q_{\nu}\right) L_{1}^{-1}$.

Proof. Since $\mathcal{H}$ has a Hamiltonian block triangular form, we have (5.2) and $\mathcal{P}$ can be easy to determined to obtain (5.3). A given $\omega$ specifies the number elements $\lambda_{k},-\bar{\lambda}_{k}$ and hence by Theorems 3.7 and 3.4 we obtain the parameterizations for the bases of the associated Lagrangian invariant subspaces of $\mathcal{H}$. Thus by Proposition 5.1 we have the conclusion.

REMARK 3. If in the Hamiltonian matrix $\mathcal{H}=\left[\begin{array}{cc}A & M \\ G & -A^{H}\end{array}\right]$ the matrix $M$ is positive or negative semidefinite, then the invertibility of $L_{1}$ in (5.4) is ensured by a controllability assumption, see Theorem 3.1 and Remark 3.2 in [9] or [15] for details. If (5.1) has an Hermitian solution with respect to a selection $\omega$, then the uniqueness follows directly from the uniqueness results for Lagrangian invariant subspaces.

TheOREM 5.3. Let $X=X^{H}$ be an Hermitian solution of (5.1) with $\Lambda(A-M X)=$ $\omega$. Then $X$ associated to $\omega$ is unique if and only if the related Hamiltonian matrix $\mathcal{H}$ has a unique Lagrangian invariant subspace associated to $\omega$. Moreover, in this case if $\omega \in \tilde{\Omega}(\mathcal{H})$ then for every selection in $\tilde{\Omega}(\mathcal{H})$ for which the associated Hermitian solutions exists, it is unique.

If the uniqueness condition for the Lagrangian invariant subspaces of $\mathcal{H}$ does not hold and if (5.1) has at least one Hermitian solution associated with a selection $\omega$, then (5.1) has infinitely many Hermitian solutions associated to $\omega$. 
Proof. The uniqueness conditions for the Hermitian solutions follows from the equivalence of i) and iii) in Theorems 4.2 and 4.3 .

If (5.1) has a solution $X$ associated to an $\omega$, following Theorem 5.2, there must be two sets of matrices $U_{1}, \ldots, U_{\mu}$ and $Q_{1}, \ldots, Q_{\nu}$ such that for

$$
L=\mathcal{S P} \operatorname{diag}\left(U_{1}, \ldots, U_{\mu} ; Q_{1}, \ldots, Q_{\nu}\right)=:\left[\begin{array}{c}
L_{1} \\
L_{2}
\end{array}\right]
$$

$L_{1}$ is nonsingular and $X=-L_{2} L_{1}^{-1}$. If the uniqueness condition for $\mathcal{H}$ does not hold then for at least one pair $\lambda_{k},-\bar{\lambda}_{k}$ or one purely imaginary eigenvalue $i \alpha_{k}$ the uniqueness condition does not hold. In the case of a pair $\lambda,-\bar{\lambda}$, by Theorem 3.7 the parameters $s_{1}, \ldots, s_{p}$ cannot be all zero. So the matrix $V$ cannot be void and satisfies (3.15) or equivalently (3.11). For every $V_{i, j}$ the associated equation is a singular Sylvester equation. So at least for the last $V_{i, j}$, say $V_{1, p}$, there are infinitely many solutions. This means that we can choose infinitely many bases which are near to a certain $U_{k}$. For the case of an eigenvalue $i \alpha$ from Theorem 3.4 again $s_{1}, \ldots, s_{p}$ cannot be all zero. So $W$ cannot be void. Since $W$ must satisfies the singular Lyapunov equation (3.8), there are infinitely many solutions. So we can also choose infinitely many bases which are near to a certain $Q_{k}$. Consequently if the uniqueness condition of $\mathcal{H}$ does not hold, then there are infinitely many bases $\tilde{L}$ of the Lagrangian invariant subspaces associated to $\omega$ such that ||$\tilde{L}-L\|<\| L_{1}^{-1} \|$, which implies that there are infinitely many Hermitian solutions corresponding to such $\tilde{L}$.

If an Hermitian solution $X$ is known then we can use it to verify the uniqueness.

Corollary 5.4. Let $X$ be an Hermitian solution of (5.1) with $\Lambda(A-M X)=\omega$. Let the columns of $\Phi_{k}, k=1, \ldots, \nu$, span the left eigenspaces of $A-M X$ corresponding to i $\alpha_{k}$. If $\Phi_{k}^{H} M \Phi_{k}$ is either positive definite or negative definite for all $k=1, \ldots, \nu$, then $\omega \in \tilde{\Omega}(\mathcal{H})$ implies that $X$ is unique. If $\omega \notin \tilde{\Omega}(\mathcal{H})$ then $X$ is unique if we have the additional condition that for every eigenvalue pair $\lambda_{k}$ and $-\bar{\lambda}_{k}$ the matrix $A-M X$ either has one of them as its eigenvalue and has a unique corresponding left eigenvector, or has both of them as eigenvalues and the corresponding left eigenvectors $x_{k}, y_{k}$ satisfy $x_{k}^{H} M y_{k} \neq 0$ for $k=1, \ldots, \mu$.

Proof. The proof follows directly from the fact that

$$
\mathcal{S}^{-1} \mathcal{H S}=\left[\begin{array}{cc}
A-M X & M \\
0 & -(A-M X)^{H}
\end{array}\right]=: \mathcal{R},
$$

where $\mathcal{S}=\left[\begin{array}{cc}I & 0 \\ -X & I\end{array}\right]$ is symplectic, and from v) in Theorems 4.2 and 4.3 .

6. Conclusion. Based on Hamiltonian block triangular forms for Hamiltonian matrices under symplectic similarity transformations we have given necessary and sufficient conditions for the existence and uniqueness of Lagrangian invariant subspaces. If the subspace is not unique, then we have given a complete parametrization of all possible Lagrangian invariant subspaces. We have then applied these results to derive existence and uniqueness results for Hermitian solutions of algebraic Riccati equations as corollaries.

\section{REFERENCES}

[1] G. Ammar, P. Benner, And V. Mehrmann, A multishift algorithm for the numerical solution of algebraic Riccati equations, Electr. Trans. Num. Anal., 1 (1993), pp. 33-48. 
[2] P. Benner, V. Mehrmann, And H. XU, A new method for computing the stable invariant subspace of a real Hamiltonian matrix, J. Comput. Appl. Math., 86 (1997), pp. 17-43.

[3] - A numerically stable, structure preserving method for computing the eigenvalues of real Hamiltonian or symplectic pencils, Numer. Math., 78 (1998), pp. 329-358.

[4] - A note on the numerical solution of complex Hamiltonian and skew-Hamiltonian eigenvalue problems, Electr. Trans. Num. Anal., 8 (1999), pp. 115-126.

[5] A. Bunse-Gerstner, Matrix factorization for symplectic QR-like methods, Linear Algebra Appl., 83 (1986), pp. 49-77.

[6] A. Bunse-Gerstner, R. Byers, and V. Mehrmann, Numerical methods for algebraic Riccati equations, in Proc. Workshop on the Riccati Equation in Control, Systems, and Signals, S. Bittanti, ed., Como, Italy, 1989, pp. 107-116.

[7] R. BYERs, Hamiltonian and Symplectic Algorithms for the Algebraic Riccati Equation, PhD thesis, Cornell University, Dept. Comp. Sci., Ithaca, NY, 1983.

[8] — A Hamiltonian QR-algorithm, SIAM J. Sci. Statist. Comput., 7 (1986), pp. $212-229$.

[9] G. FreIling, On the existence of Hermitian solutions of general algebraic Riccati equations, in Proceedings of the International Symposium on the Mathematical Theory of Networks and Systems, July 2000, Perpignan, France, to appear, 2001.

[10] G. Freiling AND G. Jank, Non-symmetric Riccati equations, Zeitschr. für Analysis und ihre Anw., 14 (1995), pp. 259-284.

[11] F. Gantmacher, Theory of Matrices, vol. 1, Chelsea, New York, 1959.

[12] G. Golub and C. Van Loan, Matrix Computations, Johns Hopkins University Press, Baltimore, third ed., 1996.

[13] M. Green And D. Limebeer, Linear Robust Control, Prentice-Hall, Englewood Cliffs, NJ, 1995.

[14] R. Horn and C. Johnson, Topics Matrix Analysis, Cambridge University Press, Cambridge, 1991.

[15] P. Lancaster And L. Rodman, The Algebraic Riccati Equation, Oxford University Press, Oxford, 1995.

[16] A. LAUB, A Schur method for solving algebraic Riccati equations, IEEE Trans. Automat. Control, AC-24 (1979), pp. 913-921.

[17] - Invariant subspace methods for the numerical solution of Riccati equations, in The Riccati Equation, S. Bittanti, A. Laub, and J. Willems, eds., Springer-Verlag, Berlin, 1991, pp. 163-196.

[18] W.-W. LIN AND T.-C. Ho, On Schur type decompositions for Hamiltonian and symplectic pencils, tech. report, Institute of Applied Mathematics, National Tsing Hua University, Taiwan, 1990.

[19] W.-W. Lin, V. Mehrmann, And H. XU, Canonical forms for Hamiltonian and symplectic matrices and pencils, Linear Algebra Appl., 301-303 (1999), pp. 469-533.

[20] V. Mehrmann, The Autonomous Linear Quadratic Control Problem, Theory and Numerical Solution, no. 163 in Lecture Notes in Control and Information Sciences, Springer-Verlag, Heidelberg, July 1991.

[21] V. Mehrmann and H. Xu, Numerical methods in control, J. Comput. Appl. Math., to appear (2000).

[22] C. Paige And C. VAn Loan, A Schur decomposition for Hamiltonian matrices, Linear Algebra Appl., 14 (1981), pp. 11-32.

[23] A. RAN AND L. Rodman, Stability of invariant maximal semidefinite subspaces, Linear Algebra Appl., 62 (1984), pp. 51-86.

[24] - Stability of invariant Lagrangian subspaces i, Operator Theory: Advances and Applications (I. Gohberg ed.), 32 (1988), pp. 181-218

[25] - Stability of invariant Lagrangian subspaces ii, Operator Theory: Advances and Applications ( H.Dym, S. Goldberg, M.A. Kaashoek and P. Lancaster eds.), 40 (1989), pp. 391-425.

[26] M. SHAYMAN, Homogeneous indices, feedback invariants and control system structure theorem for generalized linear systems, SIAM J. Cont. Optim., 26 (1988), pp. 387-400.

[27] V. Sima, Algorithms for Linear-Quadratic Optimization, vol. 200 of Pure and Applied Mathematics, Marcel Dekker, Inc., New York, NY, 1996.

[28] K. Zhou, J. Doyle, And K. Glover, Robust and Optimal Control, Prentice-Hall, Upper Saddle River, NJ, 1995. 